Kohzaki and Murakami

\title{
A transcription factor Abf1 facilitates ORC binding onto the Saccharomyces cerevisiae replication origin via histone acetylase Gen5.
}

Hidetsugu Kohzaki $£ \$ \dagger^{*}$ and Yota Murakami§£\#*

§Department of Viral Oncology, Institute for Virus Research, Kyoto University

$£$ Department of Cell Biology, Institute for Virus Research, Kyoto University

Sakyo-ku, Kyoto, Japan 606-8507

\$ Venture Laboratory, Kyoto Institute of Technology, Sakyo-ku, Kyoto, Japan 606-0951

$\dagger$ Present address: Faculty of Nursing, Shumei University, Daigaku-machi 1-1, Yachiyo,

Chiba, Japan 276-0003

\# Present address: Department of Chemistry, Faculty of Science, Hokkaido University,

Kita-ku, Sapporo, Hokkaido, Japan 060-0810

* Corresponding author: Hidetsugu Kohzaki

Department of Viral Oncology, Institute for Virus Research, Kyoto University

shogoin, Sakyo-ku, Kyoto 606-8507, Japan

E-mail: charaznable.k@gmail.com

Tel: $+81-75-723-5708$

Fax: $+81-75-723-5708$ 
Kohzaki and Murakami

9 We dedicate this work to Dr. Yasuo Kawasaki and Dr. Yuko Yamaguchi-Iwai.

Running title: Abf1 regulates the loading of ORC.

Keywords: Orc loading, chromatin structure, origin selection, transcription factor, DNA

replication, Saccharomyces cerevisiae 
Kohzaki and Murakami

\section{Abstract}

Chromatin structure has been implicated in the regulation of DNA replication but the molecular mechanism involved is unclear. In this study, we observed that binding of the transcription factor Abfl to the replication origin ARS1 facilitated the association of the origin recognition complex (ORC) with ARS1 using genetic interaction analysis and ChIP assay. The histone acetyltransferases (HATs), Gcn5 and Esa1, were also loaded onto ARS1 in an Abf1 site-dependent manner, where they were then responsible for acetylating histone $\mathrm{H} 3$ lysine 18 (H3K18) and histone H4 lysine 12 (H4K12), respectively. Interestingly, Abf1 interacted with Gcn5, while ORC interacted with Esa1. Indeed the B3 element showed genetic interaction with Gcn5 and Rpd3 not with Esa1, Act3 and Tra1.

. These data suggest that Gcn5, which is recruited by Abf1, alters chromatin structure via histone acetylation and facilitates the loading of ORC. We therefore propose that transcription factors regulate chromatin structure at replication origins by recruiting chromatin-modifying proteins, such as HATs, to load the initiator. 
Kohzaki and Murakami

\section{Introduction}

Initiation of DNA replication is highly regulated for precise genome duplication and maintenance of genome integirity. Replicon model proposed that DNA replication starts when a DNA binding protein or protein complex called an initiator binds to a defined chromosome region replicator (Jacob et al, 1964). This model seems to be applicable to almost all organisms so far analyzed. DNA replication in eukaryote cells initiates from large numbers of replication origins distributed on multiple chromosome.

In Saccharomyces cerevisiae, specific ARS (autonomously replicating sequence) element was identified on the basis of their ability to direct the autonomous replication of cloned plasmid DNA (Newlon and Theis, 1993; Shirahige, et al. 1993; Huberman 1999). ARS contains a consensus sequence (ARS consensus sequence or ACS or A element)( Newlon and Theis, 1993 ). The eukaryotic initiator protein, origin recognition complex (Orc) is first isolated from Saccharomyces cerevisiae as a six subunit ACS binding protein complex (Bell et al., 1993) and shown to be essential for the initiation of DNA replication. However, an ACS alone is not sufficient for origin function and this sequence is much more abundant than the number of the ORC binding sites or the 
Kohzaki and Murakami

functional replication origins on the genome (Marahrens and Stillman, 1992; River and Rine, 1992; Newlon and Theis, 1993; Rao et al., 1994). In addition to an ACS, many ARSs contain at least one $\mathrm{A} / \mathrm{T}$-rich region of DNA that is thought to act as a DNA unwinding element (B2 element). Though this element is important for ARS activity, it is not always required for ORC binding.

The chromosome replication cycle proceeds through multiple steps (Masumoto et al., 2000; Bell and Dutta, 2002; Bielinsky and Gerbi, 2001; Kearsey and Cotterill, 2003; Méndez and Stillman, 2003; Diffley, 2004; Cevetic and Walter, 2005; Kohzaki and Murakami, 2005; Moyer et al, 2006). The Orc is shown to stably associate with ARS during cell cycle progression. The putative replicative helicase complex, Mcm2-7 (Ishimi 1997; Shechter et al, 2004; Takahashi et al, 2005), is recruited onto Orc-bound origins with asist of Cdc6 and Cdt1 (Whittaker et al, 2000; Tanaka and Diffley, 2002) resulting in formation of pre-replicative complex (pre-RC). The pre-RC is activated by Cdc7-Dbf4 kinase (DDK) and Cdks. DDK phosphorylates the subunits of Mcm2-7 (Jares et al., 2000; Sclafani, 2000; Masai, et al., 2006; Sheu and Stillman, 2006) and Cdc45 (Jare and Blow, 2000; Zou and Stillman, 2000) leaded to changing the conformation of the complex to facilitate the loading of subsequent factors. The CDK phosphorylates Sld2 and Sld3 to form CMG (Cdc45-Mcm2-7-GINS) complex which 
Kohzaki and Murakami

leads to assemble DNA replication fork complex containing PCNA, RF-C, PR-A and replicative DNA polymerases, $\alpha, \delta$ and $\varepsilon$ ( Moyer et al, 2006).

Large body of evidence shows the correlation between transcription and DNA replication (Hyrien et al., 1995; Sasaki, 1999; Gilbert, 2001; Méchali, 2001; Lunyak et al., 2002; Schübeler et al., 2002; MacAlpine et al., 2004; Danis et al 2004; Kohzaki and Murakami, 2005). In fact, the transcription factors regulate the chromosomal DNA replication in DNA virus, yeast and mammals. In SV40 and Polyomavirus replication, transcription factors bound to the vicinity of origin stimulate initiation of DNA replication through multiple mechanisms. GAL4-VP16, Gal4, p53 and BPV E2 stimulated DNA replication through interaction with single stranded DNA binding protein, RP-A ( Kohzaki and Murakami, 2005 ) . Runx1 stimulates DNA replication by tether origin to nuclear matrix where viral DNA replication takes place ( Chen et al., 1998; Murakami et al., 2007 ) . c-Jun recruited viral initiator, Large T antigen (Tg) to replication origin for the formation of origin-Tg initiation complex (Ito et al., 1996).

In ARS1 of Saccharomyces cerevisiae, the acidic activation domains of Ga14, p53, VP16, c-Jun, BRCA1 and replication activation domain of Runx1) have been shown to regulate replication activity when they were tethered to ARS1 (Marahrens and Stillman, 1992; Li et al., 1998; Hu et al., 1999; Kohzaki et al, 1999). In our previous study 
Kohzaki and Murakami

(Kohzaki et al. 1999), we showed that transcription factors regulate the activity of the $A R S$ in a context-dependent manner, suggesting that the chromatin structure around replication origins may play an important role in replication, as has been suggested in organisms other than S. cerevisiae (Gerbi and Bielinsky 2002). In addition, it has been shown that the chromatin structure, particularly the state of histone acetylation, surrounding replication origins, can regulate the timing of origin firing (Vogelauer et al. 2002). However, the molecular mechanisms by which transcription factors or chromatin structure regulate cellular DNA replication are not yet understood(Raghuraman et al. 2001; Kim et al. 2003; Wyrick et al. 2003).

In this report, we showed that the $\mathrm{B} 3$ element of $A R S 1$ that is a binding sites for transcription factor Abfl facilitates Orc binding onto origin using ChIP assay and genetic analyses. Level of acetylation of histone H3 lysine 18 (H3K18) and histone H4 lysin 12 (H4K2), which are mark for active chromatin, was significantly decreased without B3 element. Transcription factors, LexA-VP16 and Gen4 can rescue Orc loading as well as histon actylation. Chromatin inmmunoprecipitation and genetic interaction experiments showed that Gen5 that acetylaes H3K18 associate with ARS1 through Abf1 bound to B3, while Esa1 that acetylate H4K12 located onto ARS1 through Orc. These data suggested that Abf1 recruits Gen5 to provide an active 
Kohzaki and Murakami

chromatin structure for ORC binding and then Orc recruits Esal for subsequent replication steps.

\section{Results}

To clarify the mechanism by which transcription factors regulate the activity of replication origins, we first analyzed the effect of various replication-related mutants (see Supplemental information) on to the replication of ARS1 plasmids harboring mutations in B3 or B2 element (Table S4). We prepared three B3 mutants, B3/mB3, B3/Gal4 and B3/LexA as shown in Fig.S2. In the B3 mutant, B3/mB3, the B3 element was replaced with the point mutation in ABF1 binding site (Marahrens and Stillman 1992; Kohzaki et al. 1999). In the B3 mutants, B3/Gal4 and B3/LexA, the B3 element was replaced with a Gal4 binding site and a LexA binding site, respectively (Marahrens and Stillman 1992; Kohzaki et al. 1999). We also prepared the mB2 mutant, in which B2 element is changed for Xho linker in Fig.S2. Since B3 is a binding site for transcription factor, Abf1 and B2 is corresponding to DNA unwinding element, both of which are required for efficient ARS1 plasmid replication, mutations in B2 or B3 caused instability of the ARS plasmid (Marahrens and Stillman 1992). Mutations of genes coding MCM proteins (mcm5, mcm7), DNA polymerases (pol1, cdc17), CDC28 
Kohzaki and Murakami

(cdc28as-1), GINS (dpb2, dpb11), single stranded DNA binding protein (rfa2), RFC (cdc44) and polymerase-loading protein (cdc45, JET1) increased the instability of the B2-mutant ARS1 plasmid but did not affect that of the B3 mutant plasmid. In contrast, mutations in genes coding ORC (orc1, orc2) or ABF1 (abf1) specifically increased the instability of the B3 mutant plasmid. Interestingly, mutation in of a gene coding CDC6 (cdc6-1) did not affect the instability of the B2 and B3-mutant ARS1 plasmid (Table. S4). These data showed that B3 element genetically interacts with ORC and ABF1. The genetic interaction between B2 element and various replication mutants including DNA polymerases and MCM is consistent with the notion that $\mathrm{B} 2$ element is the site where DNA is unwound and replication fork complex is formed.

Next we investigated which step in the initiation of replication is regulated by the transcription factors binding to $A R S 1$ by comparing the ability of wild-type and mutant $A R S 1$ to bind to the replication proteins using the ChIP assay. We used yeast strains bearing mutations in the $\mathrm{B} 2$ (mB2 in Fig.S2) or the B3 (B3/mB3, B3/Gal4 and B3/LexA in Fig.S2) elements of chromosomal ARS1 (Marahrens and Stillman 1992) (Fig. S2). We first analyzed the association of ORC with the chromosomal origins ARS1 and ARS305, both of which are strong ARSs (Fig. S3) by ChIP assay for tagged Orc subunits (myc-tagged ORC2 or HA-tagged ORC1) with cells arrested at the late G1, 
Kohzaki and Murakami

early $\mathrm{S}, \mathrm{G} 1 / \mathrm{S}$ or $\mathrm{M}$ phases. In all phases tested, the HA-tagged Orc1 and the myc-tagged Orc2 subunit associated with both origin sequences, but not with the non-origin sequence, $C Y C 1$ (lane 5 in each panel and S3A-E). The B3 mutation in ARS1 reduced the association of ORC with ARS1 (Fig. S3F), but not with ARS305, in the late G1, early S and M phases (lane 7 in each panel and S3G). As previously reported (Bell and Dutta 2002; Kearsey and Cotterill 2003; Méndez and Stillman 2003; Diffley 2004; Moyer et al. 2006), the Mcm complex, Cdc45, Rfa, Rfc, Pol2 and DNA polymerase $\alpha$-primase are sequentially loaded onto the origins, as shown by the timing of appearance of ChIP signals of HA-tagged Mcm4, a subunit of the Mcm complex (Fig. 1A, lane 4); HA-tagged Cdc45 (Fig. S3I), myc-tagged Rfa1, a subunit of Rfa (Fig. 1B, lane 5) HA-tagged Pol2 ( Fig. S3H) and myc-tagged Pril, a subunit of the DNA polymerase $\alpha$-primase (pol $\alpha$-primase) complex (Fig. 1C, lane 8). Loading of these proteins onto the mutant ARS1 (B3/LexA) was not detected (Fig. S3, lane 7; Fig. 1A, lane 6; Fig. 1B, lane 7; Fig. 1C, lane 10 in each panel, Fig S3), while loading onto ARS305 was unaffected in the same strain. Since the loading of thess replication factors depend on the loading of ORC (Bell and Dutta 2002; Kearsey and Cotterill 2003; Méndez and Stillman 2003; Diffley 2004; Moyer et al. 2006), these results show that the $\mathrm{B} 3$ element is of primary importance in ORC-loading onto ARS1 and that mutation 
Kohzaki and Murakami

of this element disrupts subsequent loading or replication factors. Exogenous expression of LexA-VP16 fusion protein could support ORC-loading onto ARS1 (B3/LexA), but expression of LexA DNA binding domain alone could not (Supplemental Discussion and Fig. S4). These results indicated that the primary function of transcription factors during replication is the loading of ORC onto the replication origins, which is consistent with the genetic interaction between B3 element and ORC (Figure S4).

In contrast, we found that the loading and the movment of Pri1 was delayed in the presence of the B2 mutation, although the loading of other factors (Orc2) was not affected. We also found that the loading of Cdc45, Rfa, Mcm and Pol2 delayed in the presence of the B2 mutation (Figure 1A,B and $\mathrm{S} 3 \mathrm{H}, \mathrm{I}$ ). This is consistent with these factors genetically interact with B2 element (Figure S4). These results suggest that the B2 element is involved in the formation of replication forks (see Fig. 1, Fig. S3H, I and the Supplemental Discussion).

One of the functions of transcription factors during transcriptional activation is changing the chromatin structure around promoters (Workman 2006; Shahbazian and Grunstein 2007) and the relationship between histone acetylation and active chromatin has been well documented (Workman 2006; Shahbazian and Grunstein 2007). Therefore, we examined the effect of the B3 mutation on histone acetylation around 
Kohzaki and Murakami

ARS1. The ChIP assay was performed using the anti-H4K12Ac and anti-H3K18Ac antibodies (Suka et al. 2001; Vogelauer et al. 2002) and showed that both the chromosomal and plasmid $A R S 1$ and $A R S 305$ sequences were acetylated at histone H4K12 and H4K18 in cells either growing asynchronously or blocked at late G1 by $\alpha$ -factor (Fig. 2A, lanes 5 and 11; Fig. S5, lane 5; Fig. 2B, lanes 5 and 11; Fig. 2C; Fig. S6, lane 5). Mutation of the B3 element, but not of B2, decreased the acetylation of both histones on ARS1 to undetectable levels but did not affect acetylation on ARS305 (Fig. 2A, lanes 7 and 13; Fig. S5, lane 6; Fig.2B, lanes 7 and 13; Fig. S6, lane 6). These results showed that active $A R S$ sequences are hyperacetylated, a state that represents "active chromatin" and that, in ARS1, the B3 mutation changed the acetylation state to the hypoacetylated state, which represents "inactive" chromatin.

Next, we focused on Esa1, a member of the MYST family of histone acetyltransferases, and Gcn5, as they are responsible for the acetylation of H4K12 and H3K18, respectively (Suka et al. 2001). Using a yeast strain expressing HA-tagged Esal at the same level as the endogenous Esa1 (Reid et al. 2000) (Fig. S7), we analyzed whether Esal and ARS1 interact, using the ChIP assay (Fig. 3A). Esa1 bound to ARS1 with a similar strength as it did to its other known target loci, RPS11B, RPS19B, RPS8A, RPL2B , RPL5 and RPS5 (Reid et al. 2000) (Fig. 3A) but not to the CYC1 and ACT1 
Kohzaki and Murakami

locus.

Similarly, the ChIP assay, using an anti-Gen5 antibody, indicated that Gen5 also localized to $A R S 1$, irrespective of galactose induction, which is known to cause Gen5 to bind to its target loci, such as Gall-10 (Fig. 3B; Fig. S8) (Lemieux and Gandreu 2004). Gen5 was not detected at other loci, including ARN1 or ACT1 (Fig. 3B, lanes 5 and 11; Fig. S8, lanes 5 and 9). The B3 mutation reduced the binding of GCN5 with ARS1 to undetectable levels (Fig. 3B, lanes 7 and 13; Fig. S8 lanes 6 and 10), but it did not affect the localization at Gal1-10. These data showed that Gcn5 constitutively bound to ARS1 in a B3-dependent manner.

Since HBO1, a mammalian counterpart of Esa1, was shown to interact with ORC in mammals (Iizuka and Stillman 1999; Burke et al. 2001), we investigated the interaction between ORC and Esal using an immunoprecipitation assay. When Orc2-9myc was immunoprecipitated with anti-myc (Fig. S7B lanes 5 and 6) or an anti-ORC antibody (Fig. S7B lanes 8 and 9), HA-Esa1 was co-precipitated, indicating that Esal binds to ORC. These data suggest that a complex consisting of Esal and ORC may be responsible for acetylation of H4K12 at the ARS elements.

Immuno-precipitation assay using an anti-myc antibody and strains expressing Gen5-9myc indicated that Gen5-9myc was co-precipitated with endogenous Abf1 (Fig. 
Kohzaki and Murakami

S7B, lanes 2-4 compared with lanes 10-12). Conversely, when Abf1 was precipitated by an Abf1-specific antibody, Gcn5-9myc could be detected in the precipitates (Fig. S7C, lanes 6-8 compared with lanes 14-16). These data suggest that Gen5 is recruited to ARS1 by the binding of Abf1 to the $\mathrm{B} 3$ element and Gcn5 subsequently induces the acetylation of $\mathrm{H} 3 \mathrm{~K} 18$ in the surrounding chromatin.

We also analyzed genetic interaction between $\mathrm{B} 2$ or B3 element and gcn5, esal or histone deacetylase $r p d 3$. Rpd3 is a histone deacethylase, which deacetylates all four core histones in both a global and targeted manner (Shahbazian and Grunstain, 2007). As shown in Table.S4, the B3 element showed synthetic defects gcn5 or rpd3 but not esal. On the other hands, the B2 mutant showed synthetic defect with esal but not gcn5 or rpd3. These data suggests that the level of histone acetylation regulates the ARS activity and GCN5, RPD3function through B3 element, while ESA1 functions through B3 and B2 element. Our data suggested that the chromatin around origins was modified dynamically during DNA replication, which is also suggested by Grunstein et al.

To test the function of Gen5 in DNA replication, we analyzed the stability of a plasmid bearing the Gen4-binding site instead of the B3 element (pARS1 (B3/GCN4). The transcriptional activator Gen4 is known to target Gen5 to specific sites (Kuo et al. 2000). As shown in Fig.S15, pARS1 (B3/GCN4) was more stable than the B3 mutant 
Kohzaki and Murakami

(B3/LexA), though less stable than the wild-type ARS1 plasmid. This suggests that Gcn4 can partially replace Abf1 function, probably by recruiting Gen5. We were unable to perform the stability assay using $\Delta g c n 5$ cells, because the $\Delta g c n 5$ strains could not maintain the wild-type $A R S 1$ plasmid (Table S2). This also suggests that gcn5 is important for ARS plasmid maintenance. In addition, the replacement of B3/LexA to B3/GCN4 recovered the maitotic stability defects in several strains especially in K6447 (using $\mathrm{T}$ testing, $\mathrm{p}<0.05)($ Fig.S13). Consistent with the stability assay described above, ChIP analysis indicated that Orc2 localized to pARS1 (B3/Gcn4) at the same level as the wild-type ARS1, but did not localize to pARS1 (B3/LexA or B3/mB3) (using T testing, $\mathrm{p}<0.05$ ) (Fig. 4A; Fig. S14). We found that H3K18 was acetylated around ARS1 (B3/GCN4) (Fig. S6; Fig. 4B, lane 6), but H4K12 was not (Fig. 4C, lane 6). Thus, Gcn4/Gcn5 appears to recruit ORC to the ARS, but at levels that are too low to recruit Esa1 for $\mathrm{H} 3 \mathrm{~K} 18$ acetylation.

\section{Discussion}

In this study, we showed that a transcription factor ABF1 binding to $\mathrm{B} 3$ element in ARS1 is required for efficient Orc loading onto ARS1. Abf1 recruits a 
Kohzaki and Murakami

HAT, GCN5, resulting in histone acetylation including H3K18 around ARS1. Therefore, we speculate that the change of chromatin structure induced by histone acetylation by GCN5 promotes loading of ORC (Fig S9). This speculation is further supported by the specific synthetic defects between orc mutations (orc2-1, orc1-3 and orc1-4) and B3 element mutations (mB3, B3/Gal4 and B3/LexA)

Lipford and Bell suggested that ORC could still bind to ARS1 in the B3 mutant, based on their findings with an in vivo nucleosome mapping technique that is rather indirect assay to see the ORC binding (Lipford and Bell 2001), which showed that a B3 mutation had no effect on the nucleosome positioning pattern around the A element, which represents the binding site of the ORC. However, $\mathrm{Hu}$ et al. showed that a mutation in the A element itself did not change the overall nucleosome positioning $(\mathrm{Hu}$ et al. 1999), indicating that ORC binding status does not affect the nucleosome positioning. Since it has been shown that a B3 mutation results in decreased, but still significant, replication activity of $A R S 1$, both on the plasmids and the chromosomes (Marahrens and Stillman 1992; Marahrens and Stillman 1994; Kohzaki et al. 1999), functional ORC binding to ARS1 seems to be maintained, to a certain extent, in the B3 mutant. We speculate that the absence of the B3 element/Abf1 binding destabilizes the ORC/A element association and that this can be detected by the ChIP assay, but not 
Kohzaki and Murakami

by the nucleosome mapping assay. In addition, the nature of the mutation introduced into the $\mathrm{B} 3$ element might also be responsible for the different results. In a previous report, it was suggested that Orc1 does bind to a B3-deficient ARS1(Aparicio et al.1997). However, in that study, an XhoI linker was substituted for the B3 element in the ChIP assay, while in this present study, we substituted the LexA binding site for the B3 element. In support of our assumption, we found that the extent of the synthetic growth defects in orc mutants bearing B3 mutations was influenced by the type of B3 mutation present; Lex A substitution mutants showed more severe defects compared with other mutations (B3/Gal4 and B3/mB3 ) (Fig. S4). In addition, the difference in the ORC subunit used in the ChIP assay, or the conditions of cross-linking, may have affected the sensitivity of the ChIP assay. The decreased association of other replication components (Mcm, Rfa, Cdc45, DNA polymerase 2(Pol2) and DNA polymerase $\alpha$-primase) with the B3-deficient ARS1 chromosome and plasmid (Fig. 1 and Fig. S3) further supports the requirement of the B3 element for ORC loading.

In this study, we have shown that Abf1 recruits another HAT, Gcn5, to ARS1 in order to acetylate histones (including H3K18) around ARS1. Therefore, we speculate that the change of chromatin structure induced by Gcn5-directed histone 
Kohzaki and Murakami

acetylation promotes the loading of ORC. Several lines of evidence have indicated, directly or indirectly, that chromatin structure regulates DNA replication; for example, histones located around the origins that control amplification of the chorion gene loci in Drosophila follicle cells are hyperacetylated (Aggarwal and Calvi 2004). Tethering the histone deacetylase Rpd3 to the amplification origin decreased its replication activity, whereas tethering the Hat1 homologue, Chameau acetyltransferase, increased origin activity (Aggarwal and Calvi 2004; Kohzaki and Murakami in preparation). Therefore, we assume that Gcn5, which is recruited by Abf1 to the B3 element, alters chromatin structure by acetylating histones around $A R S 1$, thereby stabilizing or enhancing the association with ORC.

We also found that Esa1 was recruited to $A R S 1$ by ORC. Esa1, a yeast histone acetyltransferase of the MYST family and its mammalian counterpart $\mathrm{HBO}$, has been linked to the ORC. HBO1 associates with pre-RC components, such as Orc1, Orc2, Mcm2, Geminin and Cdc6, in vitro and regulates the initiation of DNA replication by acetylating the pre-RC component (Iizuka and Stillman 1999; Burke et al. 2001; Iizuka et al. 2006). Sas2 has been genetically linked to the Orc2 and Orc5 subunits of ORC, though it is not yet clear whether it plays a role in regulating replication initiation (Ehrenhofer et al.1997). Recently, Kurat et al showed Gcn5 and Esa1 each contribute separately to maximum DNA synthesis rates(Kurat et al, 2017).

We speculate that Esa1, recruited by ORC, acetylates histones and probably also the components of the pre-RC to facilitate subsequent initiation steps.

The use of specific origins appears to change dramatically during development (Kohzaki and Murakami 2005; Aladjem 2007). At the onset of zygotic transcription in 
Kohzaki and Murakami

Drosophila and Xenopus, replication origins become restricted to a limited set of sites.

Also, eukaryotic replication origins exhibit different initiation efficiencies and activation timings. It is possible that the alteration of chromatin structure, induced by transcription factors, could be one of the mechanisms that regulate the timing of replication and origin selection. It is noteworthy that, in mammals, many transcription factors are also proto-oncogenes, such as c-Jun, E2F, Rb, c-Myb and c-Myc. Though, the oncogenicity of these genes is thought to be due to their involvement in the disruption of transcription, it is possible that their malfunction in the regulation of DNA replication would undoubtedly contribute to their oncogenic potential (Kohzaki and Murakami 2005; Dominguez-Sola et al. 2007). More detailed information about the inter-relationship between transcriptional regulation and DNA replication would need to be stored to understand the whole process of cell growth regulation and oncogenesis.

\section{Methods}

The Supplemental Methods section provides detailed information regarding all experimental procedures: (1) yeast strains and culture; (2) mitotic stability assay; (3) synchronization; (4) ChIP assay; (5) plasmid and yeast construction; (6) protein expression and (7) imunoprecipitation and western blotting. 
Kohzaki and Murakami

\section{References}

Aggarwal, B. D. and Calvi, B. R. 2004. Chromatin regulates origin activity in

Drosophila follicle cells. Nature 430: 372-376.

Aladjem, M. I. 2007. Replication in context: dynamic regulation of DNA replication patterns in metazoans. Nature reviews Genetics 8: 588-600.

Aparicio, A.M., Weinstein, D.M. and Bell, S.P. 1997. Components and Dynamics of

DNA replication complexes in S. cerevisiae: redistribution of mcm proteins and Cdc $45 p$ during S phase. Cell 91: 59-69.

Bell, S. P., Kobayashi, R. and Stillman, B. 1993. Yeast origin recognition complex functions in transcription silencing and DNA replication. Science 262: 1844-1849.

Bell, S. P. and Dutta, A. 2002. DNA replication in eukaryotic cells. Annu. Rev.

Biochem.71: 333-374. 
Kohzaki and Murakami

Bielinsky, A. K. and Gerbi, S. A. 2001. Where it all starts: eukaryotic origins of DNA replication. J. Cell Sci. 114: 643-651.

Burke, T. W., Cook, J. G., Asano, M. and Nevins, J. R. 2001. Replication factors MCM2 and ORC1 interact with the histone acetyltransferase HBO1. J. Biol. Chem. 276: $15397-15408$.

Chen L.F., Ito K., Murakami Y. and Ito Y. 1998. The capacity of polyomavirus enhancer binding protein $2 \mathrm{alphaB}(\mathrm{AML} 1 / \mathrm{Cbfa} 2)$ to stimulate polyomavirus DNA replication is related to its affinity for the nuclear matrix. Mol. Cell. Biol. 18; 4165-76.

Cvetic C. and Walter J.C. 2005. Eukaryotic origins of DNA replication: could you please be more specific? Semin. Cell. Dev. Biol. ; 343-53.

Danis E., Brodolin K., Menut S., Maiorano D., Girard-Reydet C. and Méchali M. 2004. Specification of a DNA replication origin by a transcription complex. Nat. Cell. Biol. $6 ; 721-30$. 
Kohzaki and Murakami

Diffley, J. F. X. 2004. Regulation of early events in chromosome replication. Cur.

Biol. 14: R778-R786.

Dominguez-Sola, D., Ying, C. Y., Grandori, C., Ruggiero, L., Chen, B., Li, M.,

Galloway, D. A., Gu, W., Gautier, J. and Dalla-Favera, R. 2007. Non-transcriptional control of DNA replication by c-Myc. Nature 448: 445-451.

Ehrenhofer-Murray, A. E., Kamakaka, R. T. and Rine, J. 1999. A role for the replication proteins PCNA, RF-C, Polymerase $\varepsilon$ and $\mathrm{Cdc} 45$ in transcriptional silencing in Saccharomyces cerevisiae. Genetics 153:1171-1182.

Ehrenhofer-Murray A. E, Rivier D. H. and Rine J. 1997. The role of Sas2, an acetyltransferase homologue of Saccharomyces cerevisiae, in silencing and ORC function. Genetics 45: 923-34.

Gerbi, S. A. and Bielinsky, A. K. 2002. DNA replication and chromatin. Cur. Opin. in Genet. Dev. 12: 243-248 . 
Kohzaki and Murakami

Gilbert, D. M. 2001. Making sense of eukaryotic DNA replication origins. Science 294: 96-100.

Hu, Y. F. Hao, Z. L. and Li, R. 1999. Chromatin remodeling and activation of chromosomal DNA replication by an acidic transcriptional activation domain from BRCA1. Genes \& Dev. 13: 637-642.

Huberman, J. A. 1999. Genetic methods for characterizing the cis-acting components of yeast DNA replication origins. Methods 18: 356-367.

Hyrien, O., Maric, C. and Méchali, M. 1995. Transition in specification of embryonic metazoan DNA replication origins. Science 270: 994-997.

Iizuka, M. and Stillman, B. 1999. Histone acetyltransferase HBO1 interacts with the ORC1 subunit of the human initiator protein. J. Biol. Chem. 274: 23027-23034.

Iizuka, M., Matsui, T., Takisawa, H. and Smith, M. M. 2006. Regulation of replication licensing by acetyltransferase Hbo1. Mol. Cell. Biol. 26: 1098-1108. 
Kohzaki and Murakami

Ishimi Y. 1997. A DNA helicase activity is associated with an MCM4, -6, and -7 protein complex. J. Biol. Chem. 272:24508-24513.

Ito K., Asano M., Hughes P., Kohzaki H., Masutani C., Hanaoka F., Kerppola T., Curran T., Murakami Y., and Ito Y. 1996. c-Jun stimulates origin-dependent DNA unwinding by polyomavirus large Tantigen. EMBO J. 15; 5636-5646.

Jacob, F., Brenner, S. and Cuzin, F. 1964. On the regulation of DNA synthesis in bacteria: the hypothesis of the replicon. Cold Spring Harbor Symp. Quant. Biol. 28: $329-345$.

Jares P. and Blow J.J. 2000. Xenopus cdc7 function is dependent on licensing but not on $\mathrm{XORC}, \mathrm{XCdc} 6$, or CDK activity and is required for XCdc45 loading. Genes \& Dev. 14; $1528-1540$.

Kearsey, S. E. and Cotterill, S. 2003. Enigmatic variations: Divergent modes of regulating eukaryotic DNA replication. Mol. Cell 12: 1067-1065. 
Kohzaki and Murakami

Kim S.M., Dubey D.D., Huberman J.A. 2003. Early-replicating heterochromatin. Genes \& Dev.17: 330-335.

Kohzaki H., Ito Y. and Murakami, Y. 1999. Context-dependent modulation of replication activity of Saccharomyces cerevisiae autonomously replicating sequences by transcription factors. Mol. Cell. Biol. 19: 7428-7435.

Kohzaki, H. and Murakami, Y. 2005. Transcription factors and DNA replication origin selection. Bioessays. BioEssays 27: 1107-1116.

Kuo, M.H., Baur, E., Struhl, K. and Allis, C. D. 2000. Gen4 activator targetes Gen5 histone acetyltransferase to specific promoters independently of transcription. Mol. Cell 6: 1309-1320.

Kurat C.F, Yeeles J.T.P, Patel, H, Early, A, Diffley, J.F.X. 2017. Chromatin Controls DNA Replication Origin Selection, Lagging-Strand Synthesis, and Replication Fork Rates. Mol Cell 65(1):117-130. 
Kohzaki and Murakami

Lemieux K. and Gandreu L. 2004. Targeting of Swi/Snf to the yeast Gall $\mathrm{UAS}_{\mathrm{G}}$ requires the mediators, $\mathrm{TAF}_{\mathrm{IIs}}$, and RNA polymerase II. EMBO J. 23: 4040-4050.

Li, R., Yu, D., Tanaka, M., Zheng, L., Berger, S. and Stillman, B. 1998. Activation of chromosomal DNA replication in Saccharomyces cerevisiae by acidic transcriptional activation domains. Mol. Cell. Biol. 18: 1296-1302.

Lipford, J.R. and Bell, S.P. 2001. Nucleosomes positioned by ORC facilitate the initiation of DNA replication. Mol. Cell 7: 21-30.

Lunyak, V. V., Ezrokhi, M., Smith, H. S. and Gerbi, S. A. 2002. Developmental changes in the Sciara II/9A initiation zone for DNA replication. Mol. Cell. Biol. 22: 8426-8437.

MacAlpine, D. M., Rodríguez, H. K. and Bell, S. P. 2004. Coordination of replication and transcription along a Drosophila chromosome. Genes\&Dev. 18: 3094-3105. 
Kohzaki and Murakami

Marahrens, Y. and Stillman, B. 1992. A yeast chromosome origin of DNA replication defined by multiple functional elements. Science 255: 817-823.

Marahrens, Y. and Stillman, B. 1994. Replicator dominance in a eukaryotic chromosome. EMBO J. 13: 3395-400.

Masai H., Taniyama C., Ogino K., Matsui E., Kakusho N., Matsumoto S., Kim J.M., Ishii A., Tanaka T., Kobayashi T., Tamai K., Ohtani K. and Arai K. 2006.

Phosphorylation of MCM4 by Cdc7 kinase facilitates its interaction with Cdc45 on the chromatin. J. Biol. Chem. 281; 39249-39261.

Masumoto, H, Sugino, A. and Araki, H. 2000. Dpb11 controls the association between DNA polymerases alpha and epsilon and the autonomously replicating sequence region of budding yeast. Mol. Cell. Biol. 20: 2809-2817.

Méchali, M. 2001. DNA replication origins: from sequence specificity to epigenetics. Nature Reviews Genetics 2: 640-645.

Méndez, J. and Stillman, B. 2003. Perpetuating the double helix: molecular machines at 
Kohzaki and Murakami

eukaryotic DNA replication origins. BioEssays 25: 1158-1167.

Moyer, S. E., Lewis, P. W. and Botchan, M. R. 2006. Isolation of the

Cdc45/Mcm2-7/GINS (CMG) complex, a candidate for the eukaryotic DNA replication

fork helicase. Proc. Natl. Acad. Sci. USA 103: 10236-10241.

Murakami Y., Chen L.F., Sanechika N., Kohzaki H. and Ito Y. 2007. Transcription factor Runx1 recruits the polyomavirus replication origin to replication factories. J. Cell. Biochem. 100; 1313-1323.

Newlon CS and Theis JF. 1993.The structure and function of yeast ARS elements.

Curr. Opin. Genet.Dev. 3; 752-8.

Newlon, C. S. and Theis, J.F. 2002. DNA replication joins the revolution: whole-genome views of DNA replication in budding yeast. BioEssays 24: 300-304.

Raghuraman M.K., Winzeler E.A., Collingwood D., Hunt S., Wodicka L., Conway A., Lockhart D.J., Davis R.W., Brewer B.J., Fangman W.L.2001. Replication dynamics of the yeast genome. Science. 294;115-21.

Rao H. Marahrens Y. and Stillman B. 1994. Functional conservation of multiple 
Kohzaki and Murakami

elements in yeast chromosomal replicators. Mol. Cell. Biol.14; 7643-51.

Reid, J.L., Iyer, V.R., Brown, P.O. and Struhl, K. 2000. Coordinate regulation of yeast ribosomal protein genes is associated with targeted recruitment of Esal histone acetylase. Mol. Cell 6: 1297-1307.

Rivier D.H. and Rine J. 1992. An origin of DNA replication and a transcription silencer require a common element. Science 256 ; 659-63.

Schübeler, D., Scalzo, D., Kooperberg, C., van Steensel, B., Delrow, J. and Groudine, M. 2002. Genome-wide DNA replication profile for Drosophila melanogaster: a link between transcription and replication timing. Nature Genetics 32: 438-442.

Sclafani, R. A. 2000. Cdc7p-Dbf4p becomes famous in the cell cycle. J. Cell Sci. 113: 2111-2117.

Shahbazian, M. D. and Grunstein, M. 2007. Functions of site-specific histone acetylation and deacetylation. Annu. Rev. Biochem. 76: 75-100. 
Kohzaki and Murakami

Shechter, D. Ying, C. Y. and Gautier, J. 2004. DNA unwinding is an Mcm complex-dependent and ATP hydrolysis-dependent process. J. Biol. Chem. 279: 45586-45593.

Sheu Y.J. and Stillman B. 2006. Cdc7-Dbf4 phosphorylates MCM proteins via a docking site-mediated mechanism to promote $\mathrm{S}$ phase progression. Mol. Cell. 24; 101-113.

Shirahige K., Iwasaki T., Rashid M.B., Ogasawara N. and Yoshikawa H. 1993. Location and characterization of autonomously replicating sequences from chromosome VI of Saccharomyces cerevisiae. Mol. Cell. Biol. 13; 5043-56.

Suka, N., Suka, Y., Carmen, A. A., Wu, J. and Grunstein, M. 2001. Highly specific antibodies determine histone acetylation site usage in yeast heterochromatin and euchromatin. Mol. Cell 8: 473-479.

Takahashi T.S., Wigley D.B. and Walter J.C. 2005. Pumps, paradoxes and 
Kohzaki and Murakami

ploughshares: mechanism of the MCM2-7 DNA helicase. Trends. Biochem. Sci. 30; $437-44$.

Tanaka, S., Umemori, T., Hirai, K., Muramatsu, S., Kamimura, Y. and Araki, H. 2007. CDK-dependent phosphorylation of Ald2 and Sld3 initiates DNA replication in budding yeast. Nature 445: 328-332.

Theis J.F. and Newlon C.S. 1994. Domain B of ARS307 contains two functional elements and contributes to chromosomal replication origin function. Mol. Cell. Biol.14; 7652-9.

Vogelauer, M., Rubbi, L., Lucas, I., Brewer, B. J. and Grunstein, M. 2002. Histone acetylation regulates the time of replication origin firing. Mol. Cell 10: 1223-1233.

Whittaker A.J., Royzman I., Orr-Weaver T.L. 2000. Drosophila double parked: a conserved, essential replication protein that colocalizes with the origin recognition complex and links DNA replication with mitosis and the down-regulation of S phase transcripts. Genes \& Dev.14; 1765-76. 
Kohzaki and Murakami

Wyrick J,J., Aparicio J.G., Chen T., Barnett J.D., Jennings E.G., Young R.A., Bell S.P., Aparicio O.M. 2001. Genome-wide distribution of ORC and MCM proteins in S. cerevisiae: high-resolution mapping of replication origins. Science 294;2357-60.

Workman, J. L. 2006. Nucleosome displacement in transcription. Genes \& Dev. 20: 2009-2017

Zou, L. and Stillman, B. 2000. Assembly of a complex containing Cdc45p, replication protein $\mathrm{A}$, and $\mathrm{Mcm} 2 \mathrm{p}$ at replication origins controlled by S-phase cyclin-dependent kinases and Cdc7p-Dbf4p kinase. Mol. Cell. Biol. 20: 3086-3096.

\section{Acknowledgements}

We dedicate this work to Dr. Yasuo Kawasaki (Osaka University, Osaka, Japan) and Dr. Yamaguchi-Iwai Yuko (Kyoto University, Kyoto, Japan). We do not know what we would have done without them during the early stages of this work. We thank B. Stillman, H. Araki, Y. Kawasaki, H. Maki, L. Graudreau, K. Struhl and J.F.X. Diffely 
Kohzaki and Murakami

for the yeast strains and the plasmids used in this study. We are also grateful to A.

Abiko, S. Nishino, K. Kamei, S. Hara, T. Uemura and M. Sugita for helpful discussions.

This work was partially supported by the Japanese Leukemia Research Fund. H.K.

was supported by a KIT VL grant and Y.M. was supported by a Grant-in-Aid for

Scientific Research on Priority Areas from the Japan Society for the Promotion of Science.

\section{Figure legends}

Figure 1. Abf1 regulates ORC loading onto ARS1.

(A) Loading of the MCM complex onto ARS1. Cells harboring HA-tagged Mcm4 (Mcm4-3HA), which is a subunit of the Mcm complex, and the ARS1 mutations indicated in the figure were synchronized with $\alpha$-factor in late G1. ChIP assays were performed with anti-HA antibodies as described in the Methods section. The experiments were repeated three times, with similar results. Yeast strains used were YHM08, YHK007, YHK008 and YHK009 (Table S2). M, molecular weight marker. $(B)$ The amounts of ARS1 WT PCR products obtained with ChIP assay relative to that obtained from mB2, $\mathrm{L}$ and $\mathrm{Lm} 2$ ( using $\mathrm{T}$ testing, $\mathrm{P}<0.05$ ) but not in ARS305 in A.

(C) Cells harboring myc-tagged $\mathrm{Rfc} 1$ (Rfc1-18myc) and the ARS1 mutations indicated 
Kohzaki and Murakami

in the figure, were synchronized at late G1 with $\alpha$-factor and released from the block at $37^{\circ} \mathrm{C}$ in the presence of $\mathrm{HU}$ in order to block the cells in the next G1 phase. Samples were collected for the ChIP assay at the indicated time points. ChIP assays were performed with anti-HA antibodies as described in the Methods section. The experiments were repeated three times, with similar results. Yeast strains used were YHM014, YHK004, YHK005 and YHK006 (Table S2).

(D) The amounts of ARS1 WT PCR products obtained with ChIP assay relative to that obtained from mB2, B3/LexA and B3/LexA, mB2(Lm2) (using $\mathrm{T}$ testing, $\mathrm{P}<0.05)$ but not in ARS305.

(E) Loading of pol $\alpha$-primase onto ARS1. (upper panel) Locations of PCR primers on the chromosome (arrows). (lower panel) Cells harboring myc-tagged Pril (Pril-9myc), which is a subunit of pol $\alpha$-primase, and the mutations indicated in $A R S 1$, were synchronized in late $\mathrm{G} 1$ with $\alpha$-factor and released from the block at $37^{\circ} \mathrm{C}$ in the presence of HU in order to block the cells in the next G1 phase. Samples were collected for ChIP assays at the indicated time points. ChIP assays were performed with anti-myc antibodies as described in the Methods section. PCR analysis using primers designed to amplify the non-origin sequence CYCl (upper panel) was performed on the precipitated DNA and the products were analyzed by agarose gel electrophoresis and 
Kohzaki and Murakami

EtBr staining. The experiments were repeated twice, with similar results. Yeast strains used were YHM07, YHK013, YHK001 and YHK002 (Table S2). M, molecular weight marker.

$(F)$ The amounts of ARS1 WT PCR products obtained with ChIP assay relative to that obtained from mB2, B3/L and B3/LexA,mB2(Lm2) (using $\mathrm{T}$ testing, $\mathrm{P}<0.05$ ) but not in ARS305.

Figure 2. Analysis of chromatin modification surrounding wild-type and mutant ARSI sequences using an anti-acetylated histone H4K12 antibody and an anti-acetylated histone H3K18 antibody.

(A) Cells harboring wild-type $A R S 1$ and the $A R S 1$ mutations indicated in the figure were either harvested as an asynchronous population, or were synchronized in late G1 with $\alpha$-factor. ChIP assays were performed with the anti-acetylated histone H4K12 antibody as described in the Methods section. PCR with primers (Vogelauer et al. 2002) specific to $A R S 1, A R S 305$, and the non-origin sequences, YCL049C (acetylated histone H4K12) and ChrVI-R TEL (unacetylated histone H4K12), were used to amplify the precipitated DNA for analysis by agarose gel electrophoresis and EtBr staining. 
Kohzaki and Murakami

The experiments were repeated twice, with similar results. Yeast strains used were YHM08, YHK007, YHK008 and YHK009 (Table S2). M, molecular weight marker.

(B) The amounts of ARS1 WT PCR products obtained with ChIP assay relative to that obtained from mB2, B3/LexA and B3/LexA, mB2(Lm2) but not in ARS305 of (A).

(C) Cells harboring wild-type $A R S 1$ and the mutations indicated in the figure were either harvested as an asynchronized population or were synchronized in late G1 with $\alpha$-factor. ChIP assays were performed with anti-acetylated histone H3K18 antibody, as described in the Methods section. PCR with primers (Vogelauer et al. 2002) specific to $A R S 1, A R S 305$, the non-origin sequences, ARS1412-930bp (acetylated histone H3K18) and ChrVI-R TEL (unacetylated histone H3K18), were used to amplify the precipitated DNA for analysis by agarose gel electrophoresis and EtBr staining. The experiments were repeated twice, with similar results. Yeast strains used were YHM08, YHK007, YHK008 and YHK009 (Table. S2).

(D) The amounts of ARS1 WT PCR products obtained with ChIP assay relative to that obtained from L but not in ARS305 of (C).

Figure 3. Esa1 and Gen5 are loaded onto ARS1. 
Kohzaki and Murakami

(A) ChIP assay of 3HA-Esa1. ChIP assays were performed with anti-HA, as described in the Methods section. The indicated promoters of ribosomal proteins, ARS1 and CYC1 were detected. The experiments were repeated twice, with similar results. The yeast strain used was YHK013 (Table S2). M, molecular weight marker.

(B) The amounts of the indicated promoters of ribosomal proteins, ARS1, CYC1 and ACT1 PCR products obtained with ChIP assay relative to that obtained from WCE.

(C) Cells harboring wild-type ARS1 and the mutations indicated in the figure were cultured in YPD (-Gal) or YPGal (+Gal). ChIP assays were performed with anti-Gen5, as described in the Methods section. PCR with primers (Lemieux and Gandreu 2004) specific to $A R S 1$, the non-origin sequences Gall-10 (to which Gen5 is known to localize), $A C T 1$ and $A R N 1$ (to which Gen5 never localizes) were used to amplify the precipitated DNA for analysis by agarose gel electrophoresis and EtBr staining. The experiments were repeated twice, with similar results. Yeast strains used were YHM08, YHK007, YHK008 and YHK009 (Table S2). M, molecular weight marker. (D) The amounts of ARS1 PCR products obtained with ChIP assay relative to that obtained from WCE in the presence and the absence of Galactose in C.

Figure 4. Partial rescue of the $\mathrm{B} 3$ mutation in ARS1 by Gen4. 
Kohzaki and Murakami

(A) Plasmid ChIP assay of the Gen4 site. The yeast strain K6447, harboring the plasmids indicated in the figure, was grown in SCM-Ura+Ade for the mitotic stability assay. ChIP assays were performed with anti-myc, as described in the Methods section. PCR with primers specific to the pARS1 plasmid, ARS305 and CYC1were used to amplify the precipitated DNA for analysis by agarose gel electrophoresis and EtBr staining. M, molecular weight marker.

(B) The amounts of ARS1 PCR products obtained with ChIP assay relative to that obtained from L and Gen4 but not in ARS305 in A.

(C) Plasmid ChIP assay of the Gen4 site. The yeast strain K6447, harboring the plasmids indicated in the figure, was grown in SCM-Ura+Ade for the mitotic stability assay. ChIP assays were performed with anti-acetylated histone H3K18, as described in the Methods section. PCR with primers specific to the pARS1 plasmid, ARS1412-920bp and ChrVI-R TEL were used to amplify the precipitated DNA for analysis by agarose gel electrophoresis and EtBr staining. M, molecular weight marker. (D) The amounts of ARS1 PCR products obtained with ChIP assay relative to that obtained from L and Gen4 but not in ARS305 in C.

(E) Plasmid ChIP assay of the Gen4 site. The yeast strain K6447, harboring the plasmids indicated in the figure, was grown in SCM-Ura+Ade for the mitotic stability 
Kohzaki and Murakami

assay. ChIP assays were performed with anti-acetylated histone H4K12, as described in the Methods section. PCR with primers specific to the pARS1 plasmid, ARS305, YCLO49 and ChrVI-R TEL were used to amplify the precipitated DNA for analysis by agarose gel electrophoresis and EtBr staining. The experiments were each repeated twice, with similar results.

(F) The amounts of ARS1 PCR products obtained with ChIP assay relative to that obtained from L and Gen4 but not in ARS305 in E. 
Kohzaki Fig. 1A

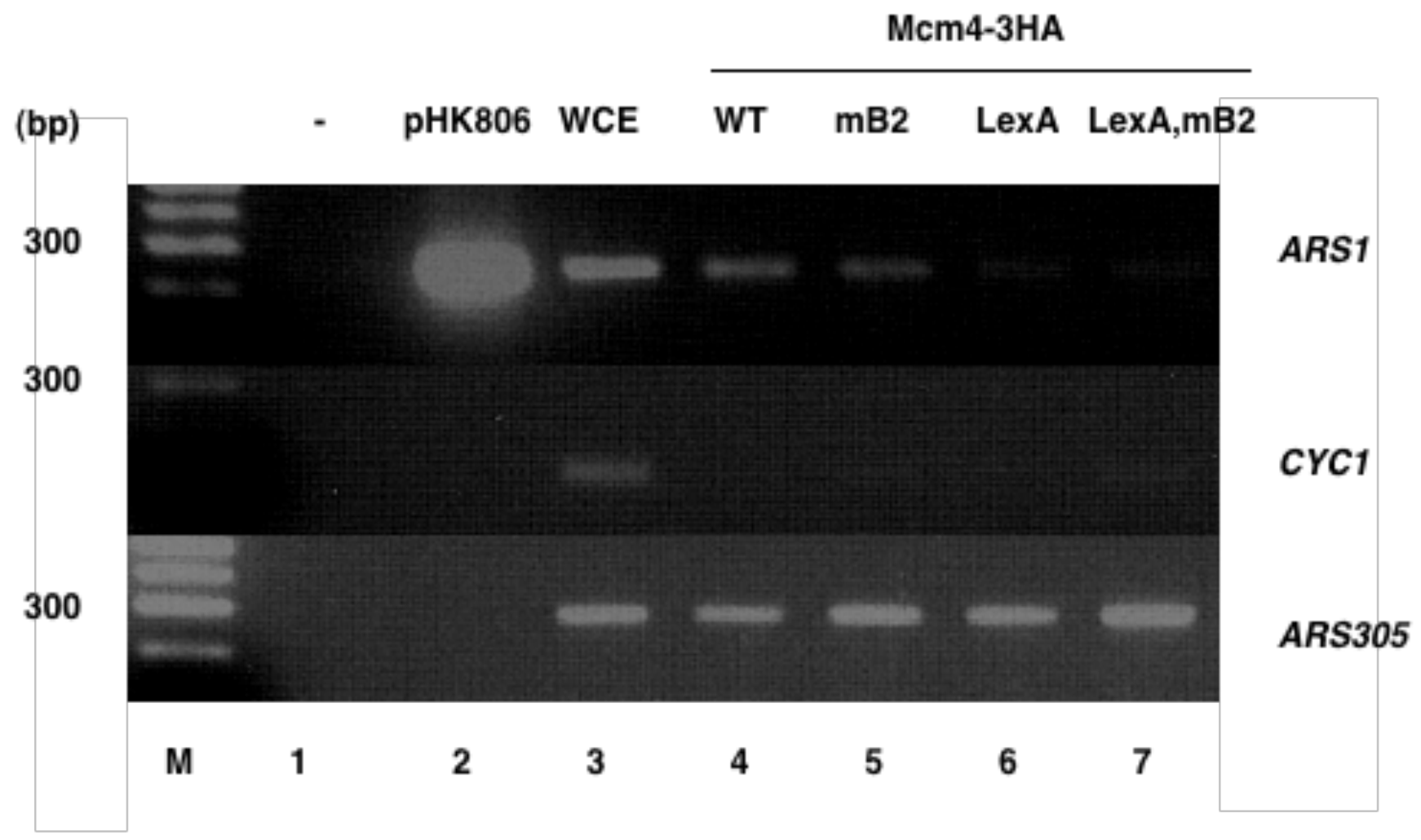




\section{Kohzaki Fig. 1B}

ARS1

Loading of the Mcm4 onto ARS1 and ARS305

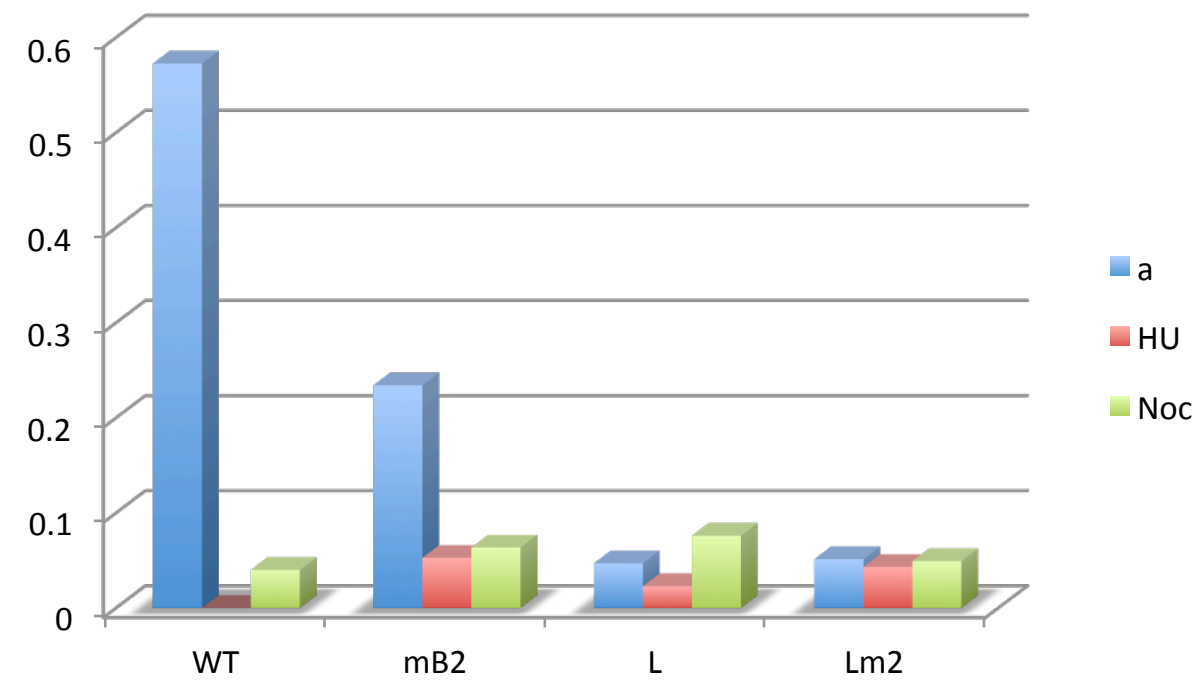

ARS305

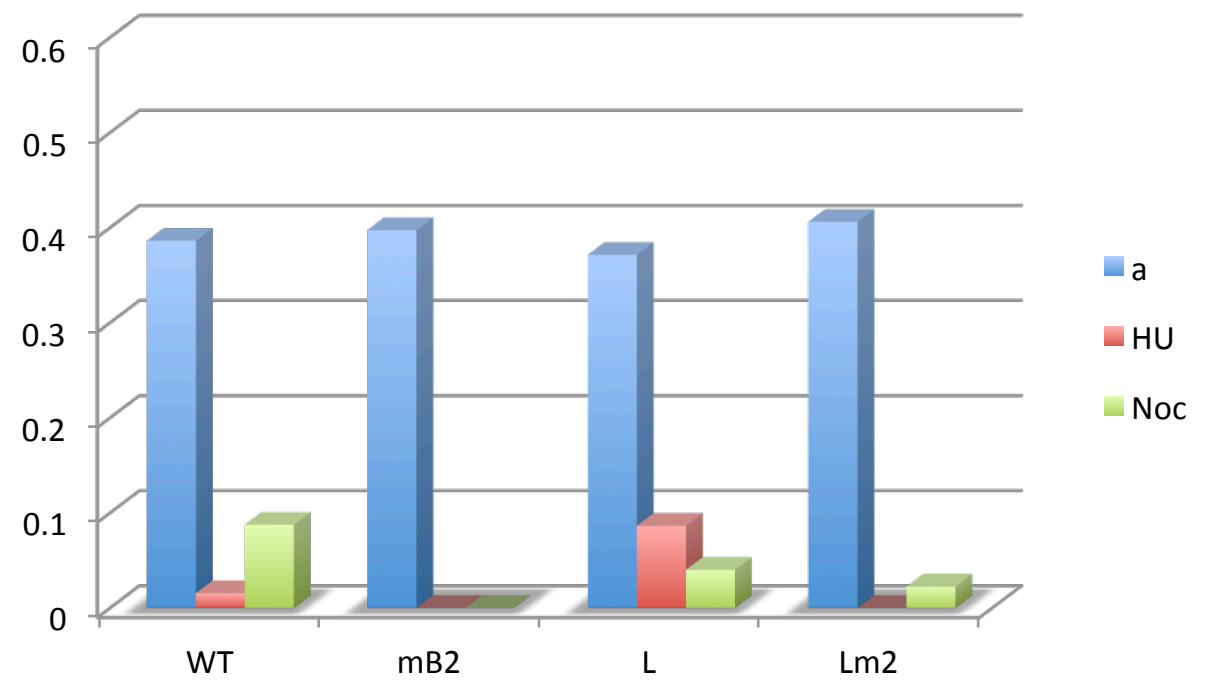



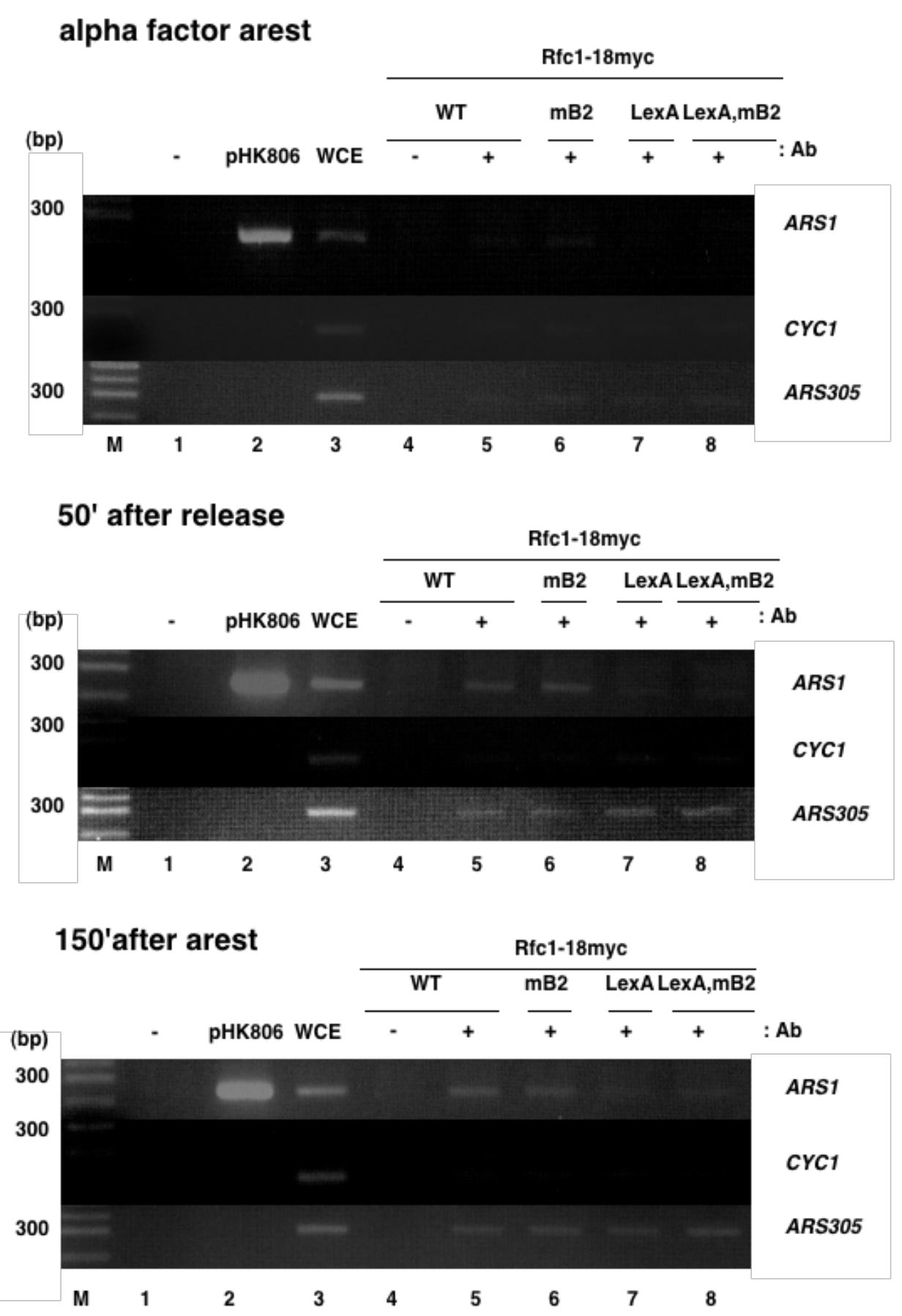


\section{Kohzaki Fig. 1D}

\section{Loading of the Rfa onto ARS1 and spreading out from ARS1}

WT
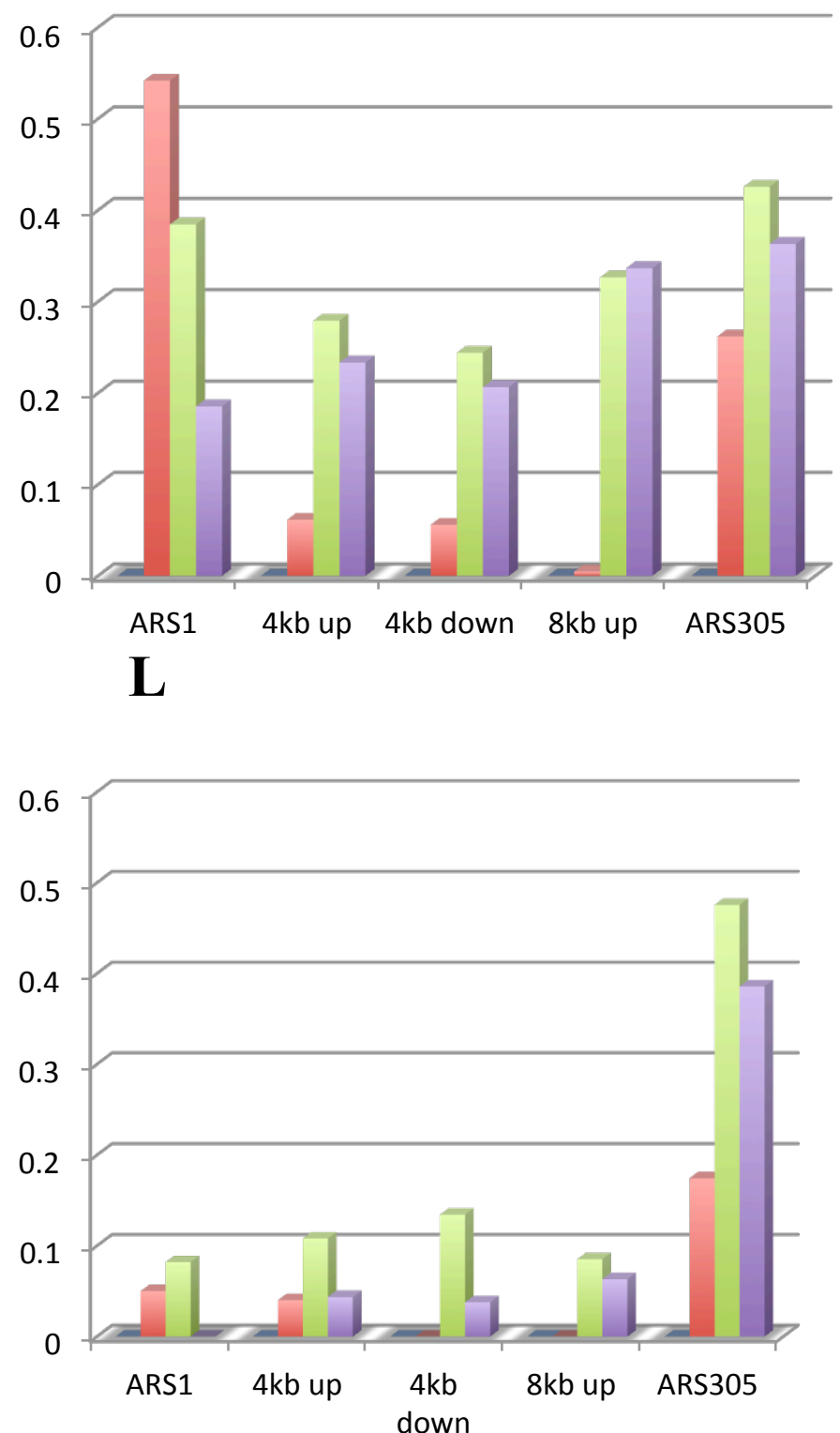

mB2

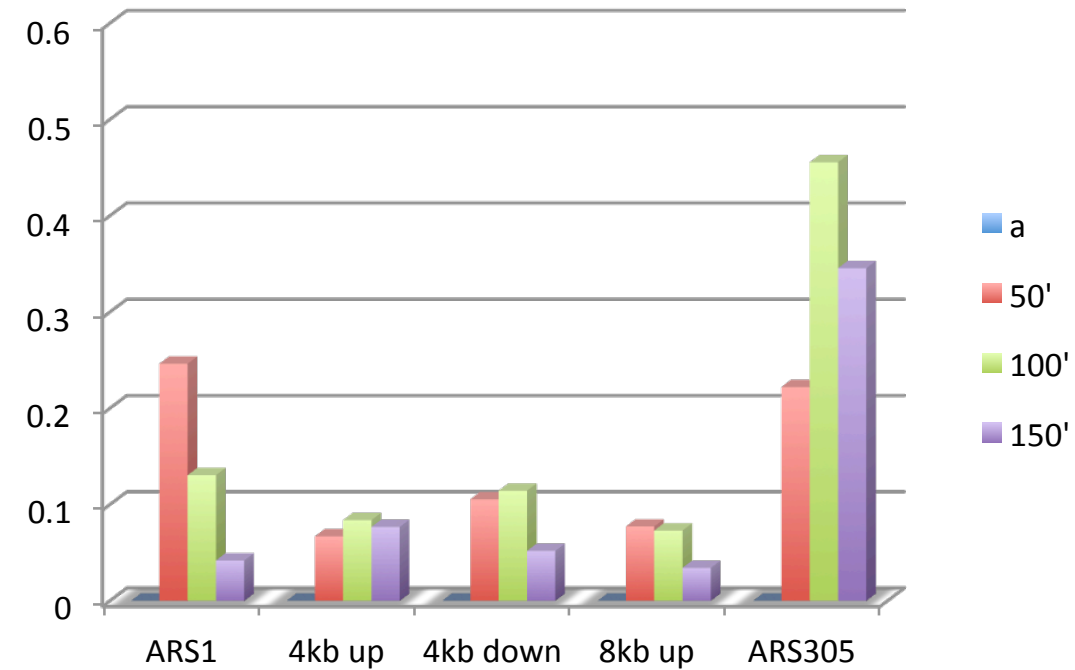

\section{Lm2}

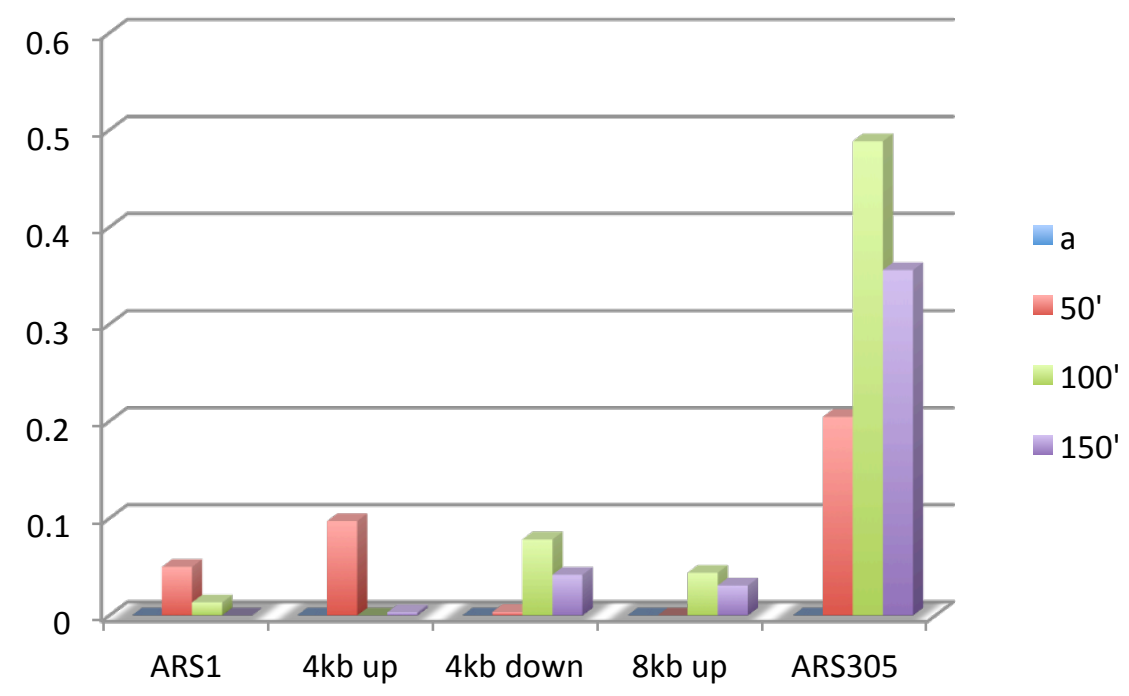


Kohzaki Fig. 1E

\section{Primer design}

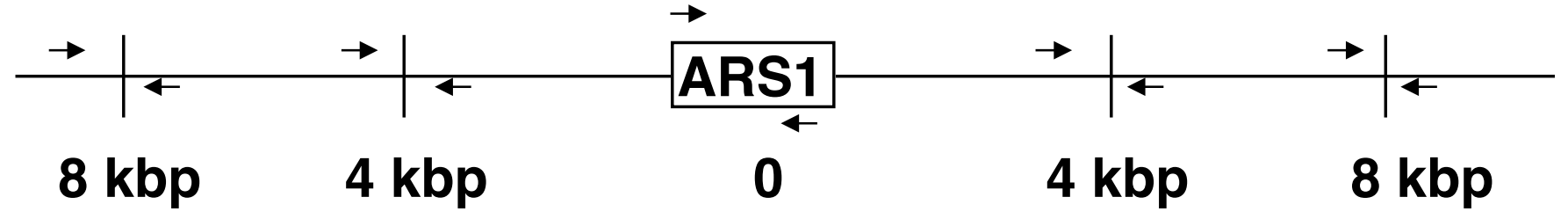

$\alpha$-factor block in the presence of $\mathrm{HU}$

$\alpha$-factor block release 165 ' in the presence of HU
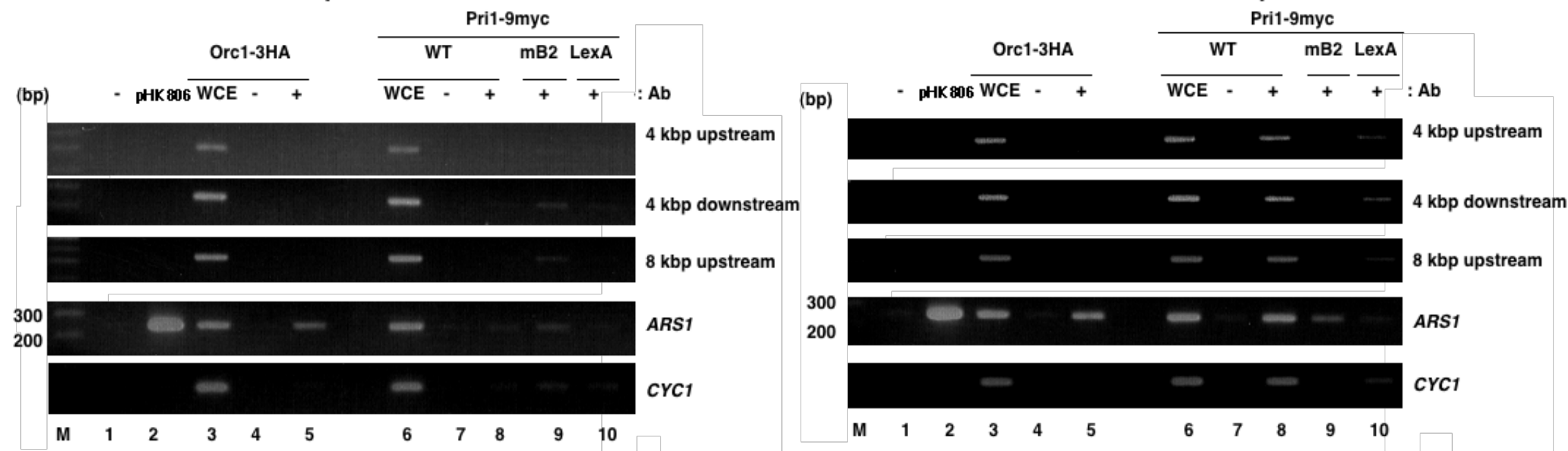

$\alpha$-factor block release $120^{\prime}$ in the presence of $\mathrm{HU}$

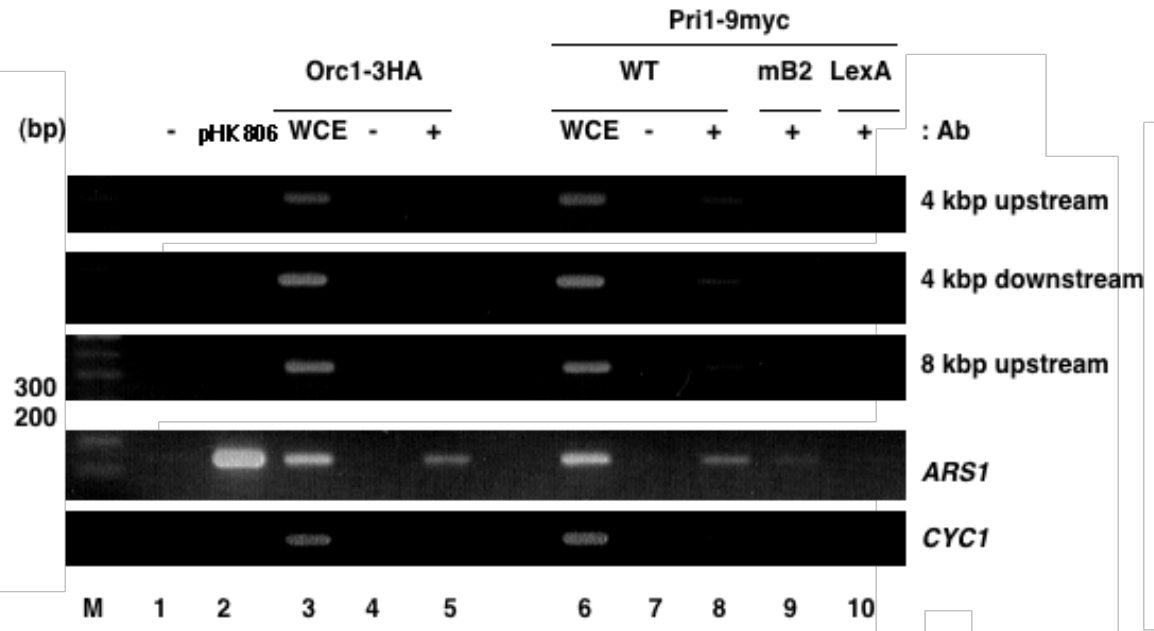

$\alpha$-factor block release 225 ' in the presence of $\mathrm{HU}$

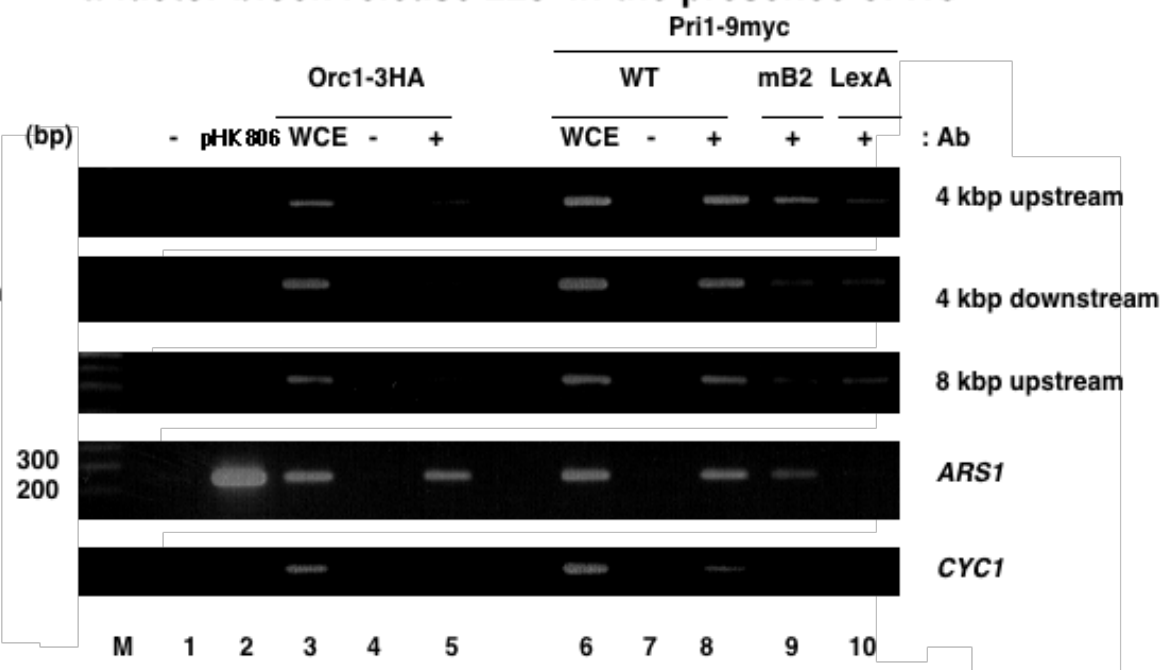




\section{Loading of the Pold-primase onto ARS1}

\section{ARS1 WT}

$\mathrm{mB} 2$
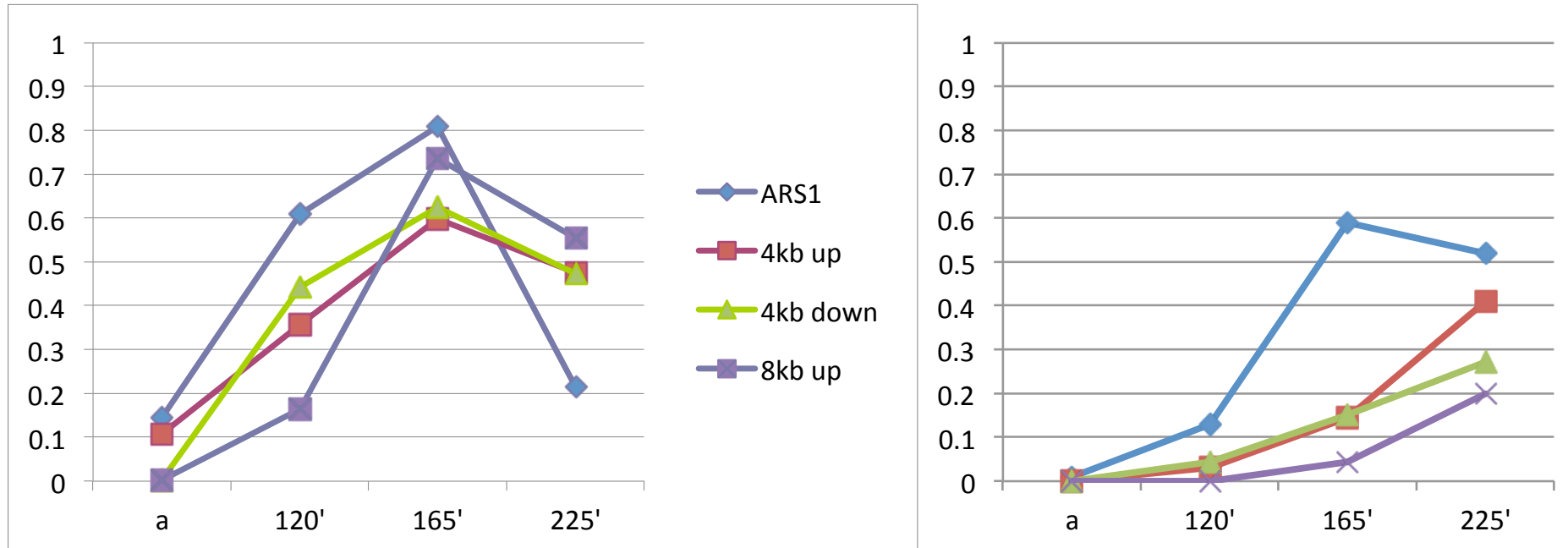

$\sim$ ARS1

$-4 k b$ up

$-4 k b$ down

$\leftarrow 8 k b$ up

$\mathrm{L}$

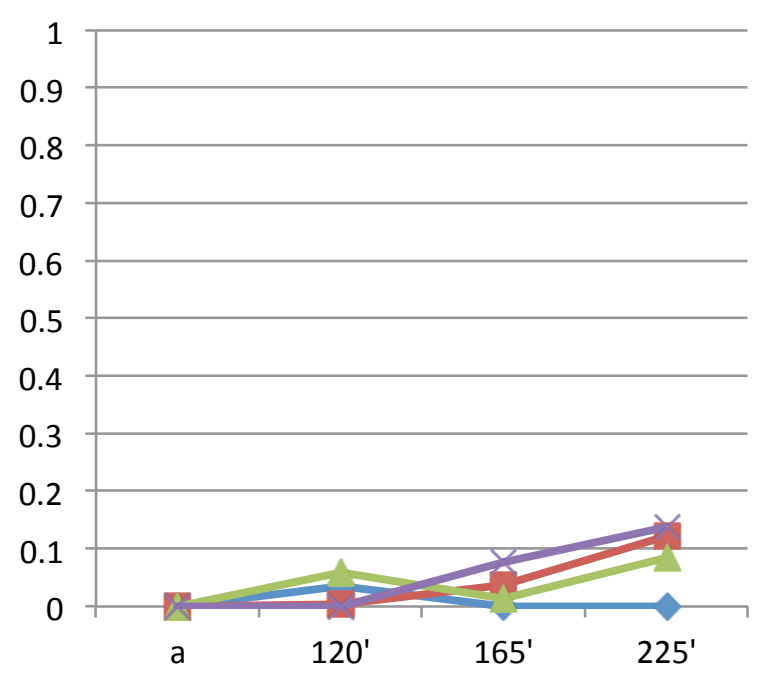

$\leadsto$ ARS1

$-4 \mathrm{~kb}$ up

$-4 \mathrm{~kb}$ down

$\leftarrow$ - $\mathrm{kb}$ up

Lm2
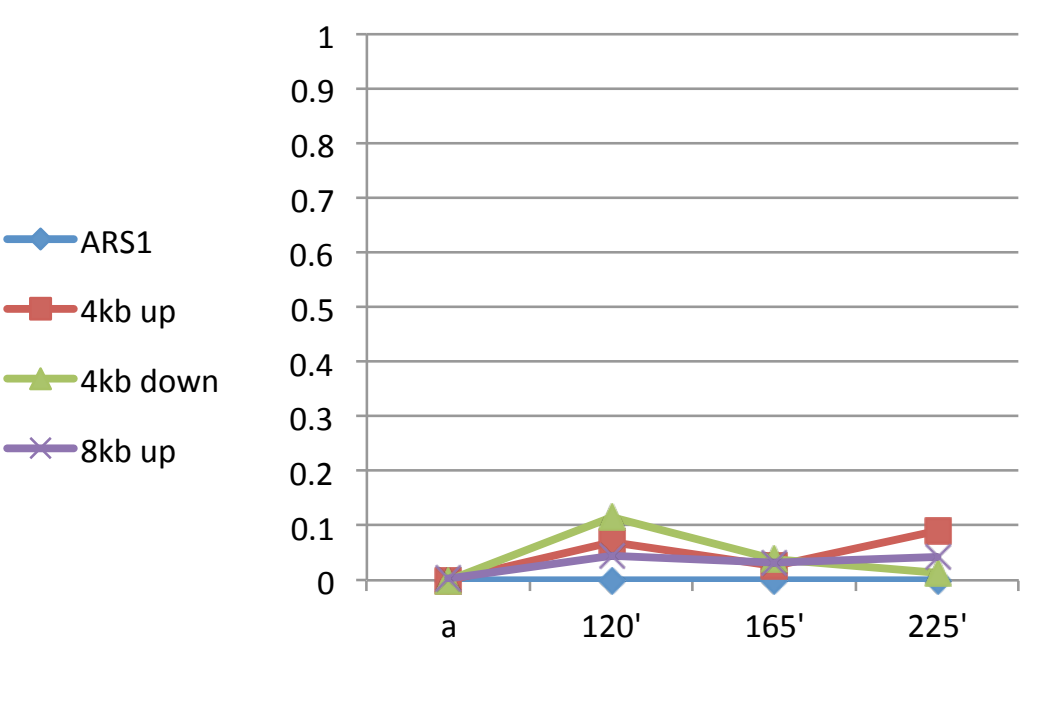
Kohzaki Fig. 2A

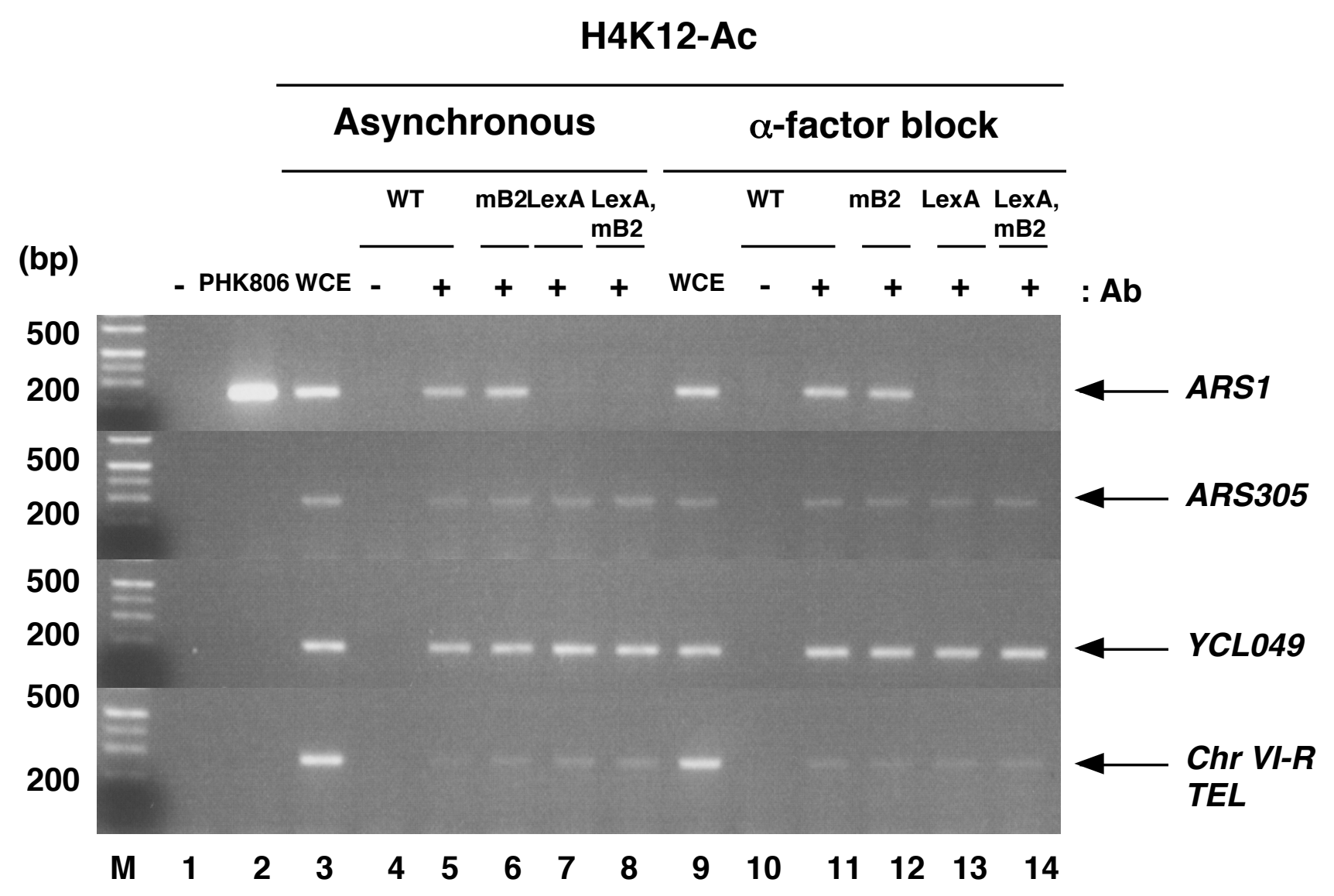




\section{Kohzaki Fig. 2B}

\section{Acetylation of HistonH4K12 around ARS1}

Asynchronous

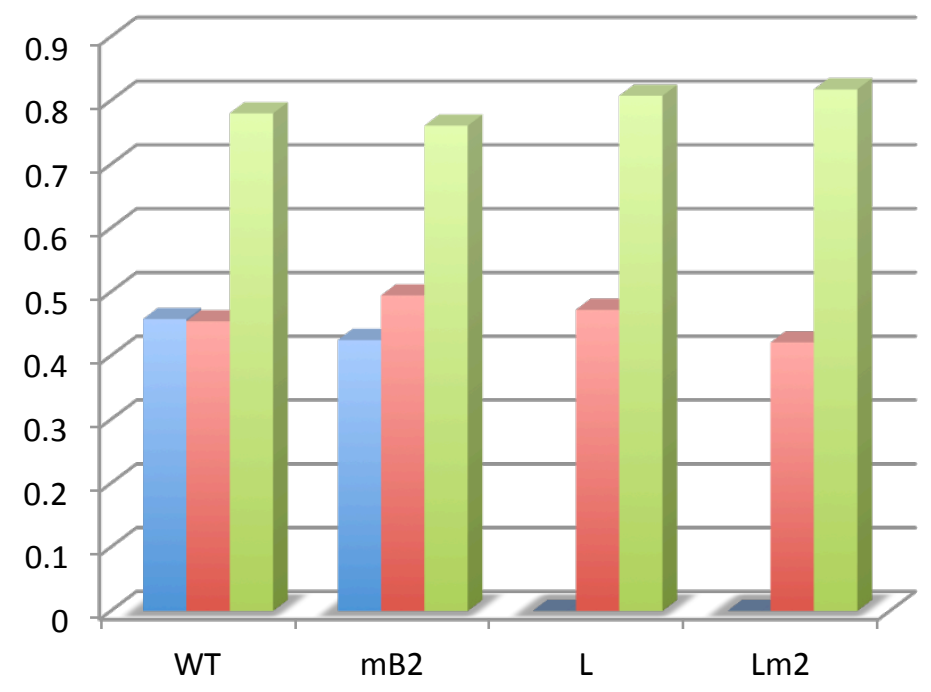

$\alpha$-factor




Kohzaki Fig. 2C

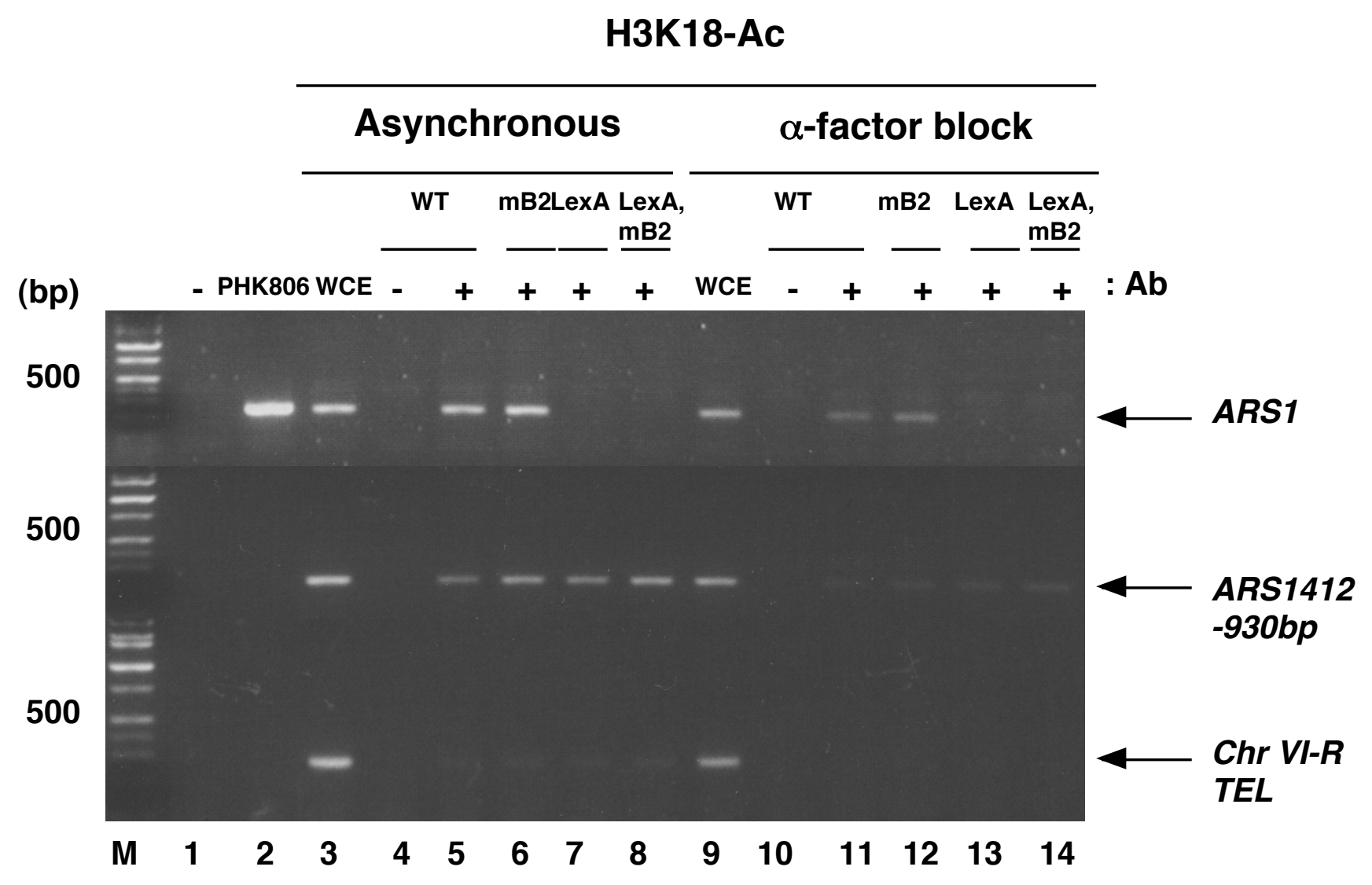




\section{Kohzaki Fig. 2D}

\section{Acetylation of HistonH3K18 around ARS1}
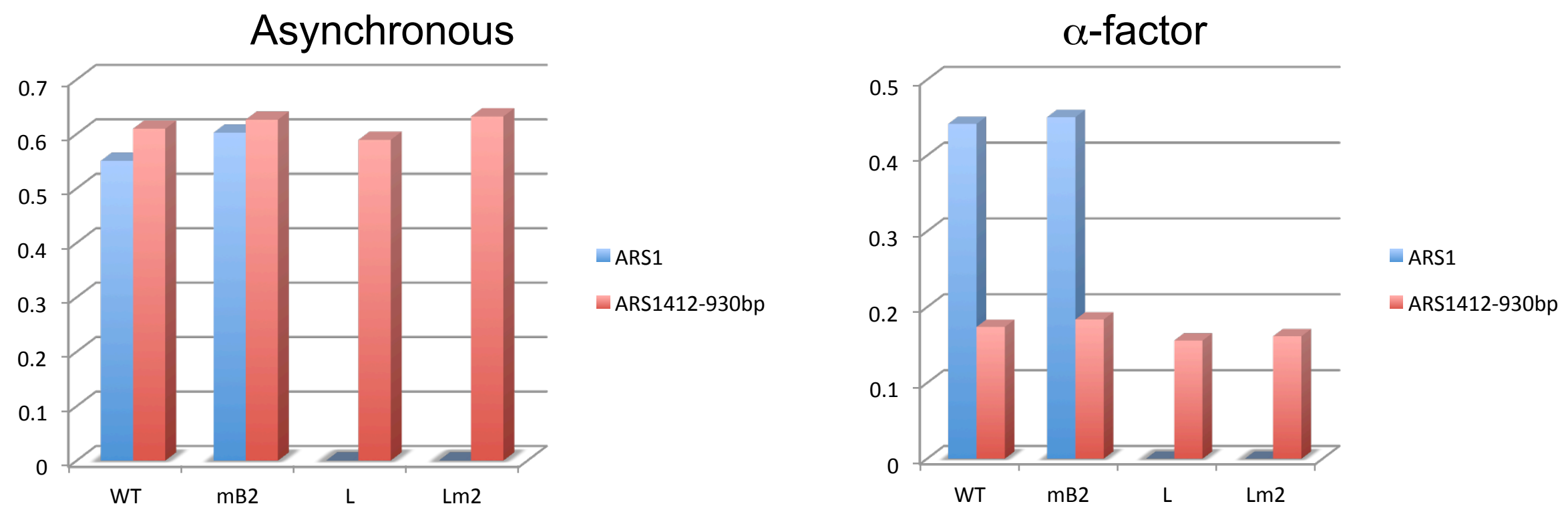


\section{Kohzaki Fig. 3A}

3HA-Esa1

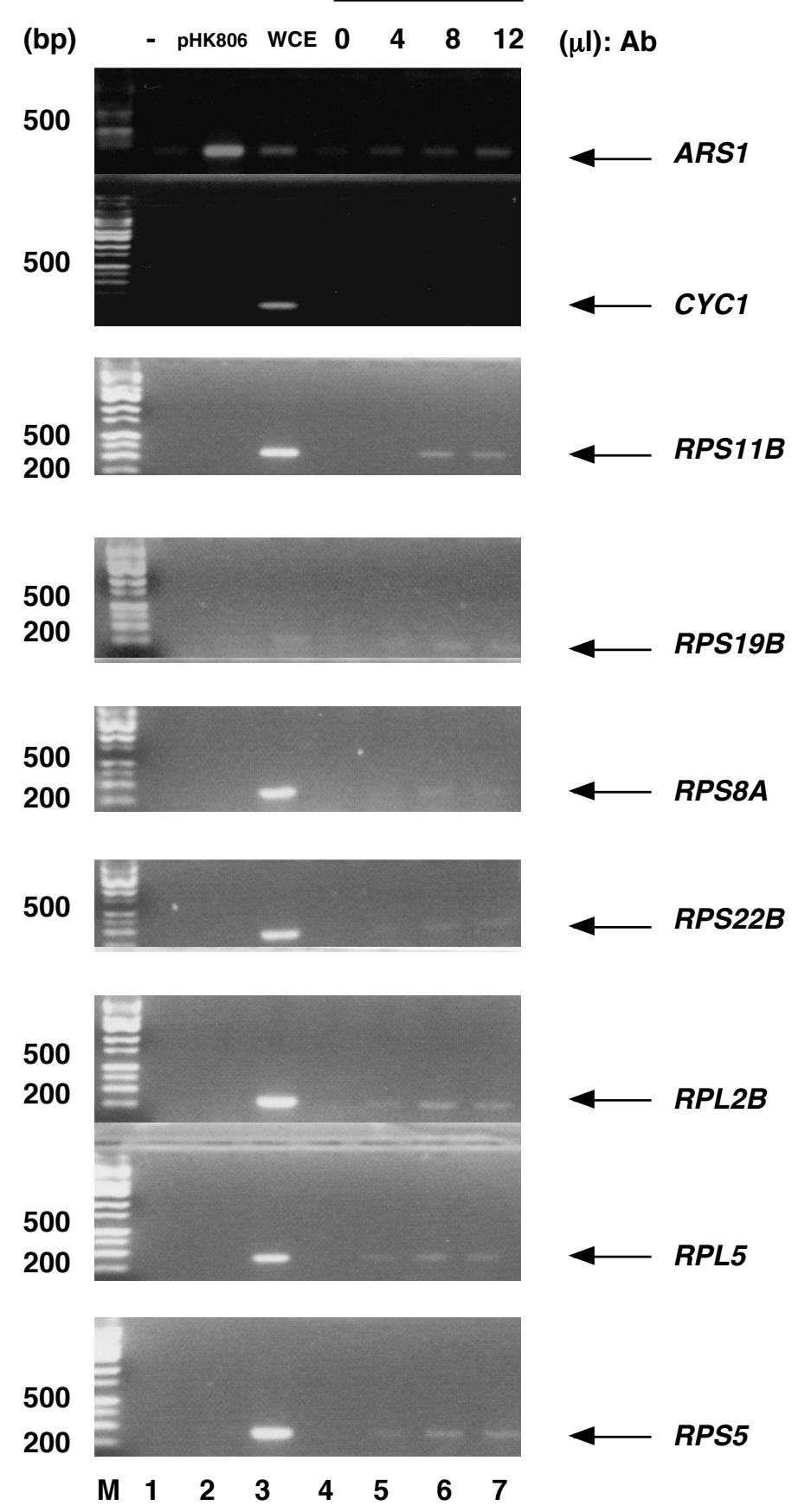




\section{Kohzaki Fig. 3B}

The Loading of Esa1 onto ARS1, Ribosomal protein promoter but not CYC1 and ACT1




Kohzaki Fig. 3C

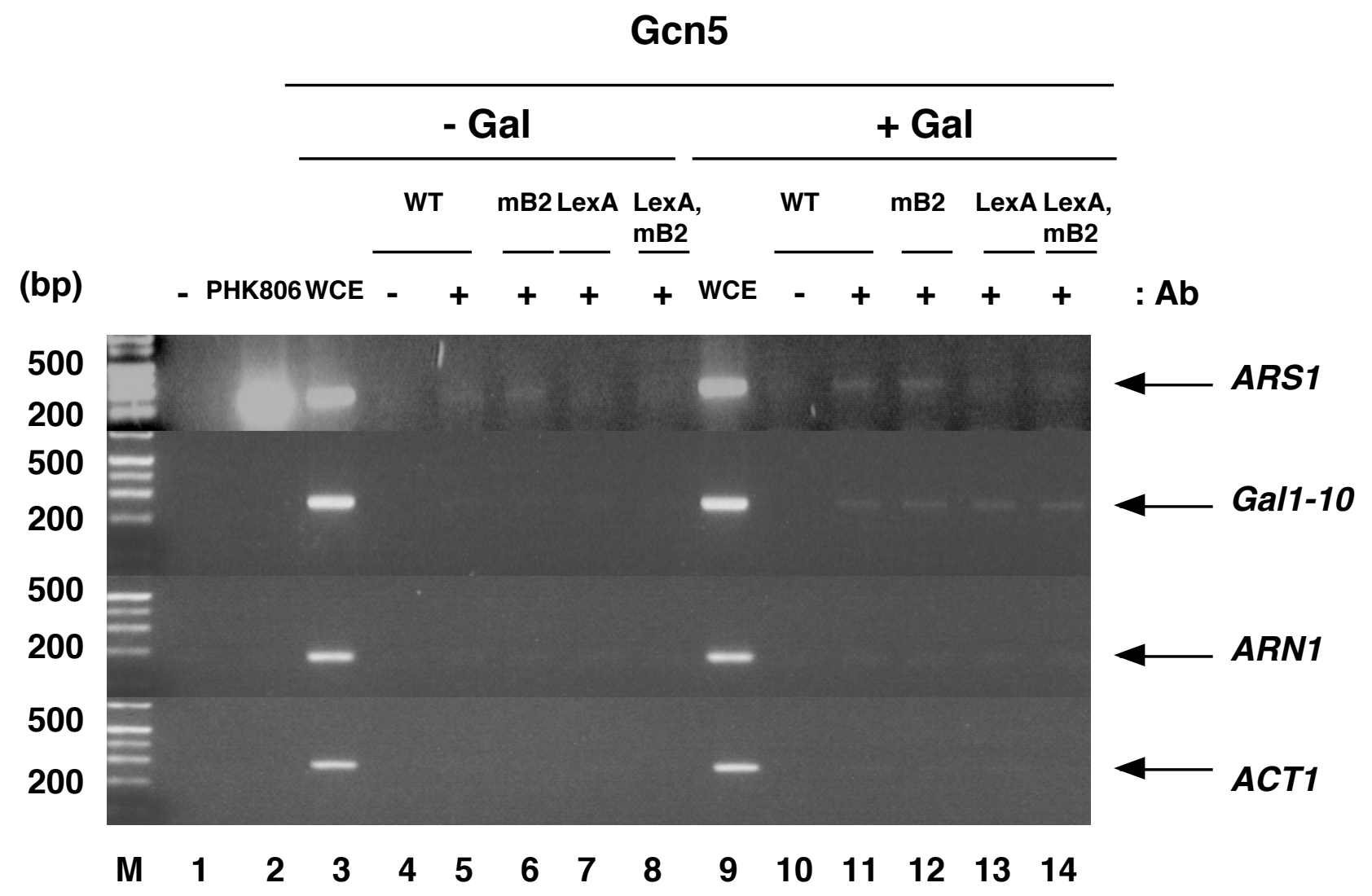




\section{Kohzaki Fig. 4}

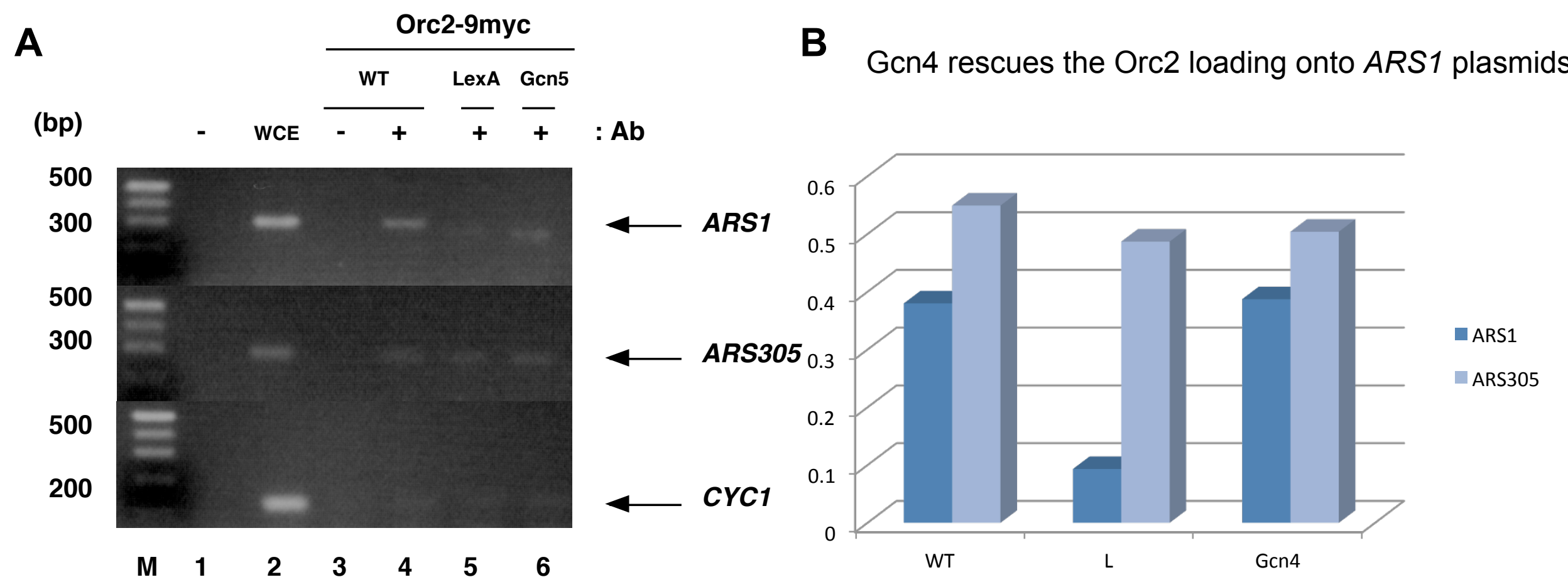




\section{Kohzaki Fig. 4}

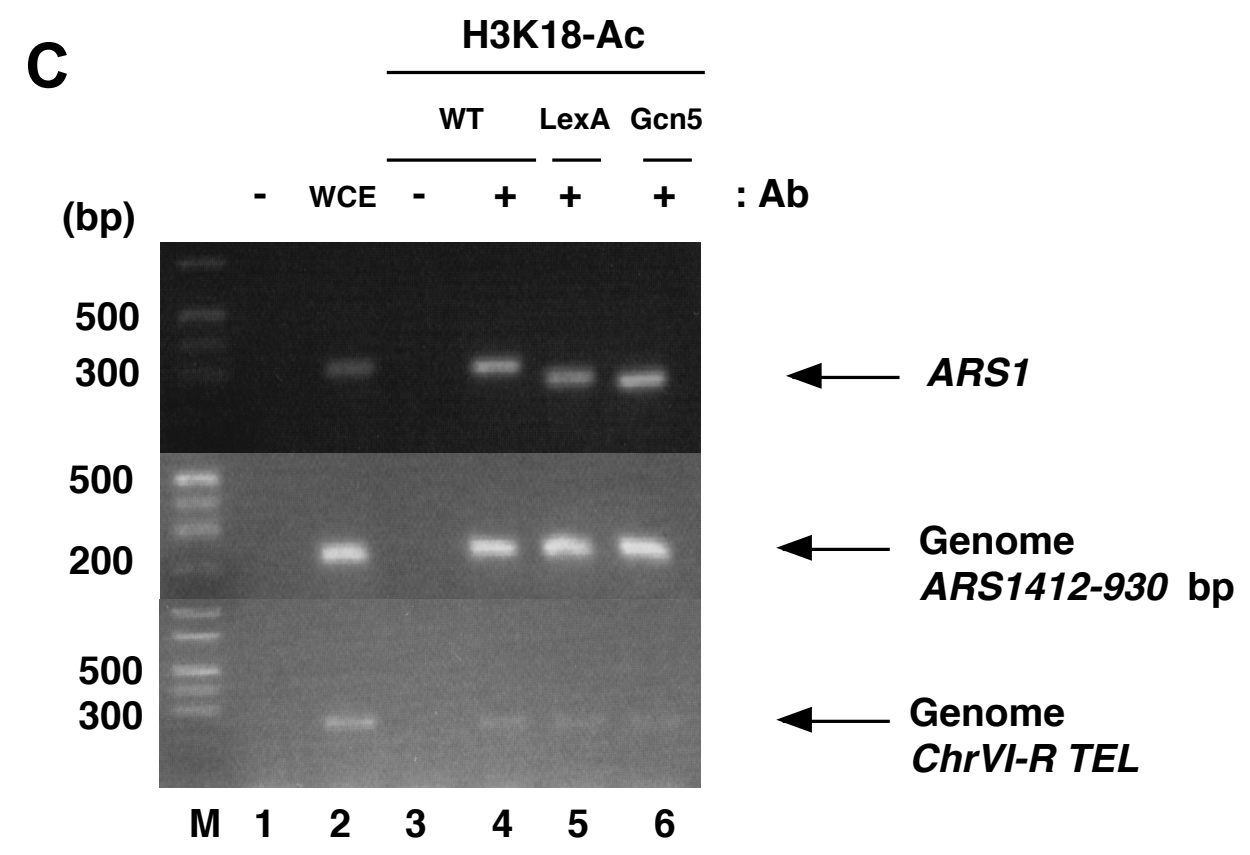
D Gcn4 rescues the acetylation of $\mathrm{H} 3 \mathrm{~K} 18$ onto ARS1 plasmids

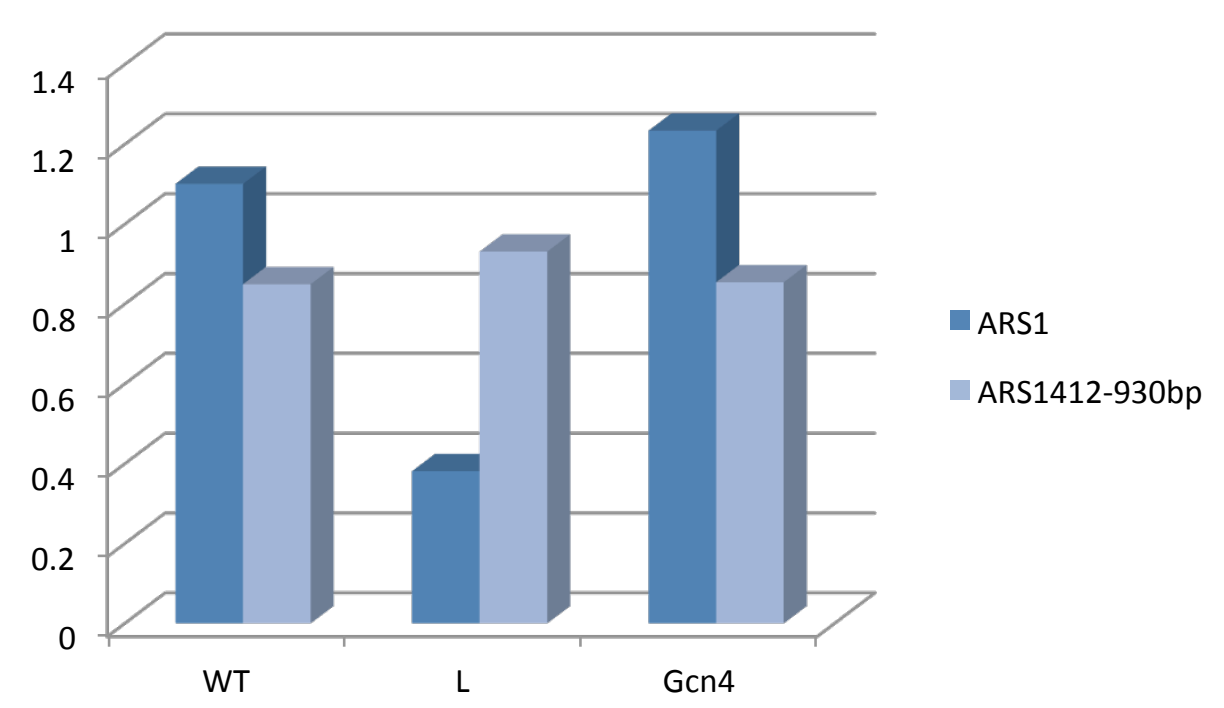




\section{Kohzaki Fig. 4}

E

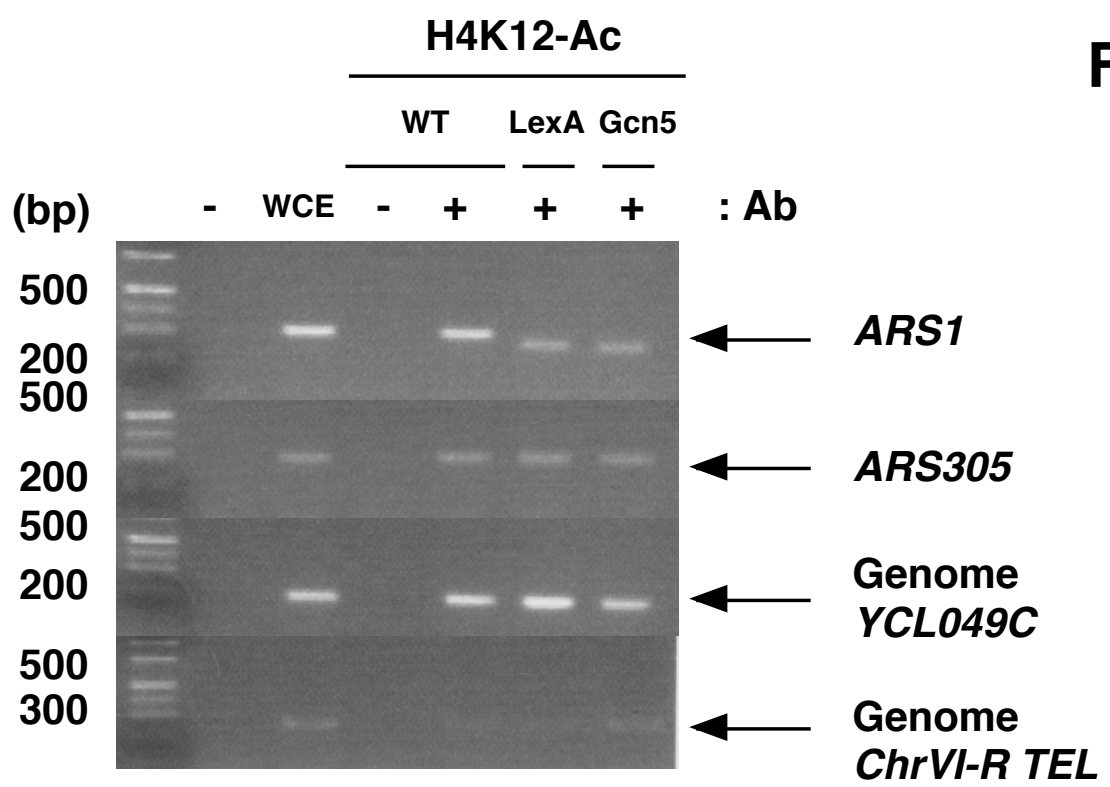

ChrVI-R TEL
F Gcn4 don't rescues the acetylation of H4K12 onto ARS1 plasmids

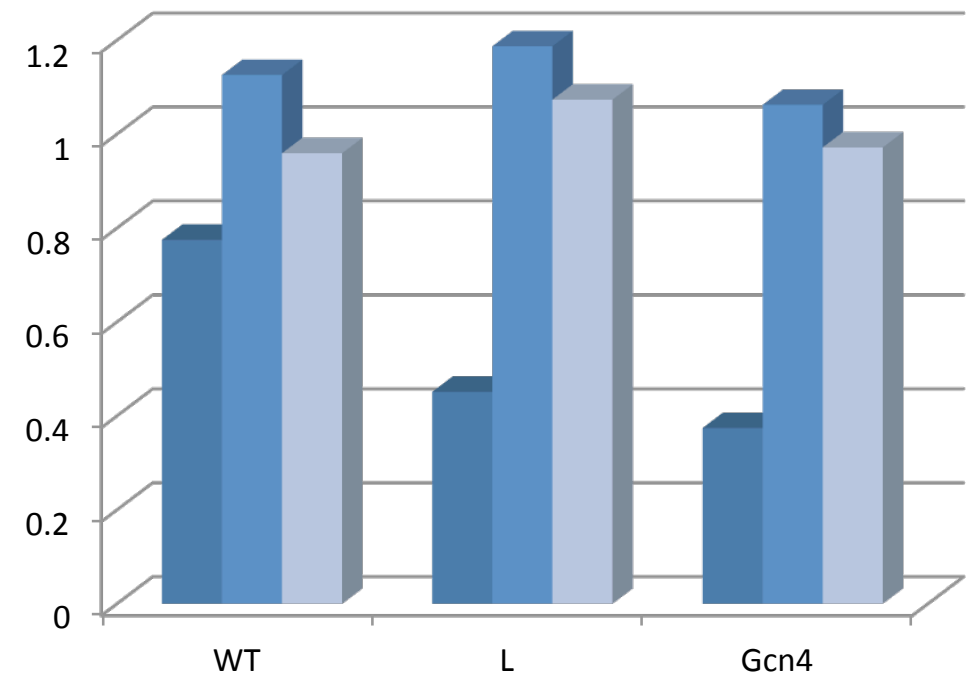

ARS1

ARS305

YCL049C

$$
\text { - }
$$

